\title{
Optimization of CubeSat System-Level Design and Propulsion Systems for Earth-Escape Missions
}

\author{
Sara Spangelo* \\ California Institute of Technology, Pasadena, California 91109 \\ and \\ Benjamin Longmier \\ University of Michigan, Ann Arbor, Michigan 48109 \\ DOI: $\underline{10.2514 / 1 . A 33136}$
}

\begin{abstract}
Small spacecraft can now perform trajectory maneuvers resulting in significant orbit changes that were once only feasible with larger spacecraft due to the development of miniaturized propulsion technology. This paper addresses the feasibility of using CubeSat Ambipolar Thruster, a large $\Delta V$ miniaturized propulsion system for constellation control, Earth escape, and planetary flybys, to enable Earth-escape maneuvers on a CubeSat form factor. Operational and trajectory variables include the power setting during thrust maneuvers, when to thrust, and the attitude control inputs. The dynamic energy available through the maneuvers, which is constrained by the power available from the sun depending on the orbit and is consumed in propulsion, is modeled as well as attitude control maneuvers and realistic battery degradation. To explore the design space of this capability, the sensitivity of solutions to spacecraft mass, fuel quantity, initial orbit, solar power collection, and battery size is demonstrated. Optimal orbit-raising techniques are compared and the optimal approach depending on the goals is discussed (i.e., minimize time, minimize fuel, minimize batteries, minimize propulsion system volume, and minimize accumulated radiation). The models and results presented lay the groundwork for future work in integrated vehicle and operational design optimization problems with both interplanetary and constellation architectures.
\end{abstract}

\begin{tabular}{lll} 
& \multicolumn{1}{c}{ Nomenclature } \\
$a$ & $=$ & acceleration \\
$e$ & $=$ & stored energy capacity \\
$g$ & $=$ & gravitational acceleration \\
$I_{\mathrm{sp}}$ & $=$ & specific impulse \\
$L$ & $=$ & length \\
$m$ & $=$ & vehicle mass \\
$\dot{m}$ & $=$ & mass flow rate \\
$p$ & $=$ & power \\
$r$ & $=$ & radius from the center of the Earth \\
$t$ & $=$ & time \\
$V_{\mathrm{ex}}$ & $=$ exhaust velocity \\
$v$ & $=$ & orbit velocity \\
$\Delta V$ & $=$ & change in velocity after a maneuver \\
$\delta$ & $=$ & mass flow rate to power ratio \\
$\mu$ & $=$ & Earth's gravitational constant
\end{tabular}

\section{Introduction}

$\mathrm{C}$ UBESATS are no longer constrained to low Earth orbits (LEOs) where they drift passively and deorbit. They now have the potential to escape Earth orbit control over their destinations and lifetimes due to innovations in small satellite propulsion systems, as well as communication system, electronics, and attitude control. This is enabling small spacecraft platforms to perform far more interesting exploration and science missions. In the past, CubeSats have not had propulsion systems due to size, power, and launch constraints and were therefore constrained to minimal maneuvering from the launch vehicle insertion orbit [1]. First, we assess the feasibility and limitations of using CubeSats for these applications, considering size,

Received 8 September 2014; revision received 27 December 2014; accepted for publication 7 January 2015; published online 20 April 2015. Copyright $\odot 2014$ by Sara Spangelo . Published by the American Institute of Aeronautics and Astronautics, Inc., with permission. Copies of this paper may be made for personal or internal use, on condition that the copier pay the $\$ 10.00$ per-copy fee to the Copyright Clearance Center, Inc., 222 Rosewood Drive, Danvers, MA 01923; include the code 1533-6794/15 and $\$ 10.00$ in correspondence with the CCC.

*Systems Engineer, Jet Propulsion Laboratory, 4800 Oak Grove Drive.

${ }^{\dagger}$ Assistant Professor, Aerospace Engineering, 1320 Beal Avenue. mass, power, and other system-level concerns that constrain the ability of small spacecraft to escape Earth orbit. Second, we introduce and compare diverse orbit-boosting approaches to achieve different objectives, for example propellant-optimal, time-optimal, or solutions that minimize the exposure to radiation subject to realistic small spacecraft constraints.

CubeSats have evolved from educational tools to a platform for technology demonstrations and are capable of performing high-value science missions [2-4]. The National Science Foundation has a dedicated program funding nanosatellites to study space weather $[5,6]$; NASA has a dedicated launching opportunity through their CubeSat Launch Initiative [7]; and NASA research centers are also developing a variety of CubeSats for both near-Earth [8,9] and interplanetary [10-13] applications. An increasing number of CubeSats are being proposed for constellation applications $[14,15]$, where propulsion capabilities are required or highly desired. Micropropulsion has been identified as a necessary technology to enable these spacecraft to maneuver and perform formation flying, create constellations, and travel to interesting interplanetary destinations $[\underline{16}, 17]$. Orbit transfers, particularly to interplanetary destinations, are particularly challenging for small spacecraft lowthrust propulsion systems because typical high-thrust maneuvers cannot be used, and small spacecraft are extremely mass-, volume-, and power-constrained [1]. Furthermore, CubeSats do not typically operate at the high power or high voltage levels required to support propulsion systems, nor do they have solutions for managing the resulting high thermal loads (most CubeSats use passive thermal control techniques).

Solar electric propulsion (SEP) has been proposed and used for a variety of interplanetary mission architectures, including optimized trajectories to the Mars surface for scientific exploration, Discoveryclass mission applications, and asteroid belt missions [18-21]. SEP was first demonstrated for interplanetary spacecraft applications on NASA's Deep Space One (DS1) mission and gathered the first in situ measurements of ion propulsion-induced plasma environment [22]. DS1 paved the way for NASA's Dawn mission powered by the Xenon Thruster, which enabled its exploration to the asteroid Vesta and the dwarf planet Ceres [23]. Optimal trajectories for interplanetary travel to asteroid swarms have been investigated considering energy dynamics for low-thrust systems [24]. Recent work has demonstrated the applicability of electric propulsion solutions for operational 
responsiveness in LEO and provided global reach requirements and optimal time and propellant solutions as well as testing out five other advanced technologies [25].

Existing electric propulsion systems exceed the mass or volume envelopes for CubeSats, have low efficiency or low thrust, or are designed for precise pointing maneuvers. The CubeSat Ambipolar Thruster (CAT) is a new thruster technology that uses a high-density plasma source to achieve high $\Delta V$ and high thrust-to-power ratios and is designed for use in CubeSat missions. The CAT engine will change the CubeSat paradigm from drifters to explorers by providing $11 \mathrm{~km} / \mathrm{s}$ delta-V to a $3 U$ CubeSat, or $20 \mathrm{~km} / \mathrm{s}$ to a $6 \mathrm{U}$ CubeSat. Our team has a dedicated NASA launch to test this thruster technology in space for the first time, and we will be collaborating with the University of Michigan and NASA Ames Research Center [7].

The CAT technology has a wide power range and thus can $\bar{b}$ e used to achieve different thrust levels throughout the trajectory. The desired thrust level at a given time is a tradeoff between the thrust level, duration of the maneuver, and available energy at that point in the trajectory, which is a function of the instantaneous power from solar arrays and energy that is stored in the battery. Minimizing transfer time or propellant use is an optimization problem with a set of highly interdependent decision variables related to energy dynamics and constrained by the battery capacity, solar panel size, and other system-level constraints.

Past work has demonstrated representative mission scenarios for a $3 \mathrm{U}$ CubeSat the feasibility of escaping Earth orbit with the conventional constant-thrust strategy that results in spiral-out trajectories using the CAT thruster [26]. Follow-on work compared conventional constant-thrusting spiral-out approaches to optimal thrusting near perigee [27] and applied and compared these strategies when performed by different CubeSat-class thrusters [28]. Spangelo and Longmier $[26,27]$ considered system-level constraints such as volume, mass, power management, and radiation exposure, and they determined that the optimal approach depends greatly on the system objectives and constraints.

There are other propulsion systems that have been proposed for CubeSats and small spacecraft, including Aerojet's Rocketdyne, Busek's thrusters, Massachusetts Institute of Technology's ion Electrospray Propulsion System, Clyde Space's CubeSat Pulse Plasma Thruster, and the Jet Propulsion Laboratory's Micro Electrospray Propulsion thruster [1]. Most of the existing electric propulsion systems have been optimized for maximum specific impulse and/or maximum efficiency. However, in our work, we show that, by taking a systems-level approach to overall spacecraft and mission optimization, parameters such as thrust-to-power ratio and impulse density are usually more import in thruster design than a higher specific impulse or higher thruster efficiency. The techniques presented in this paper are applicable to these other technologies, although they are not specifically studied in this paper.

The goal of this paper is to evaluate the feasibility and identify key tradeoffs for small spacecraft with emerging propulsion capabilities that perform orbit-boosting maneuvers. To ensure relevance of proposed solutions to realistic mission architectures, a systems-level perspective is introduced that includes CubeSat mass, volume, and power limitations as well as operational constraints. We develop an analytic model that includes propulsion, orbit dynamics, energy dynamics (solar powered collection, eclipse), battery capacity dynamics (cycling, depth of discharge, degradation), and accumulated radiation dosages. The model is used to study trajectories starting in LEO and escaping Earth's sphere of influence (SOI). Different orbit-boosting schemes are considered in this work, including constant thrusting to achieve the conventional spiraling trajectories as well as schemes that consider thrusting only during the orbits perigee with optimized thrust levels and durations. We model, simulate, optimize, and compare different orbit-raising strategies to identify the best approaches for different mission goals. Sensitivity analyses are performed relative to spacecraft mass, collected power, and battery capacity to quantify key tradeoffs in vehicle and mission design parameters and to identify feasible mission architectures.

\section{Mission and System Architecture}

\section{A. Small Spacecraft Limitations}

CubeSats are typically launched as secondary payloads into LEOs with altitudes ranging from 350 to $900 \mathrm{~km}$ and with near-polar inclinations (greater than $60 \mathrm{deg}$ ) [29]. Therefore, to demonstrate Earth-escape trajectories, we discuss orbits that begin in $500 \mathrm{~km}$ polar and equatorial orbits are considered in our analysis. There are also some opportunities for some sun-synchronous LEOs, where the combination of altitude and inclination result in the object in orbit ascending or descending over any Earth latitude at the same local mean solar time every day. In these orbits, the orbital plane is nearly orthogonal to the vector to the sun, and so the spacecraft is nearly always in the sun (i.e., experiences short or no eclipses). These orbits are also considered in the analysis to demonstrate their advantages. The general approach presented in this paper is applicable to a much broader class of missions, including spacecraft initially launched into geostationary orbit (GEO) or geostationary transfer orbit (GTO), where significantly lower $\Delta V$ is required to escape Earth orbit. If a small spacecraft can be launched as a secondary payload into one of these orbits, it will be able to escape Earth orbit considerably faster or with less power.

Small spacecraft, and specifically CubeSats, are extremely massand volume-constrained. For example, the most common CubeSat size, a $3 \mathrm{U}$, is constrained to the form factor of approximately $30 \times 10 \times 10 \mathrm{~cm}$, and usually constrained to a mass of less than $5 \mathrm{~kg}$, particularly to satisfy the standard Poly-PicoSatellite Orbital Deployer (P-POD) launcher system. Satisfying conventional P-POD launch constraints may not be a requirement for all CubeSats in the future; for example, mass waivers allowing heavier small spacecraft systems may be allowed. However, a lighter system is always desirable for orbit boosting because more acceleration is achieved for the same thrust. The desire for a low-mass system drives the selection of the thruster, propellant tank, propellant, solar panels, attitude control system, and battery and power system components, as well as indirectly the operational modes of the system.

CubeSats are constrained in their ability to collect, store, and distribute power. Typical CubeSats that have flown in LEO with body-mounted solar panels generate less than $10 \mathrm{~W}$, whereas spacecraft with state-of-the-art deployable panels and the ability to continually point them at the sun may be able to generate up to $30 \mathrm{~W}$ [30]. Furthermore, onboard CubeSat battery systems typically store only 50-100 kJ (14-30 W · h), and existing CubeSat electric power systems (EPSs) typically operate with low voltage and power values (e.g., less than or equal to $8 \mathrm{~V}$ and 10-20 W) [30]. Thus, although collecting power and storing the energy for high-powered shortduration thrusts may be feasible, supplemental battery and power management systems may be required to support these types of maneuvers (which require additional volume, mass, cost, and complexity). In addition, typical lithium-ion batteries that fly on CubeSat systems experience significant battery degradation throughout their operation, limiting their ability to support multiple hundred cycles on longer-duration missions [27].

Radiation is often a concern for CubeSat missions because the lowcost commercial off-the-shelf (COTS) components they fly are typically not radiation-tolerant, are thus susceptible to failures, and cannot reliably support long-duration missions. Mission trajectories that avoid the radiation belts as much as possible, either by operating at polar (instead of equatorial) inclinations or by boosting their orbits to high altitudes quickly to avoid radiation exposure are preferred. Developing fault-tolerant failures and adding radiation shielding is also an option; however, this may add system complexity, mass, and cost.

\section{B. Representative Vehicle Design}

Toward assessing the feasibility of achieving Earth escape with a CubeSat form factor, we consider a $3 \mathrm{U}$ CubeSat form factor [31]. 3U CubeSats are typically constrained to a mass of $5 \mathrm{~kg}$, although waivers can be obtained such that up to $7 \mathrm{~kg}$ can be accommodated. A representative list of components, mass, volume, and power, is provided in Table $\underline{1}$. where all components are included except 
Table 1 MEL for representative $3 \mathrm{U}$ CubeSat $\left(1 \mathrm{U}\right.$ is $\left.1000 \mathrm{~cm}^{3}\right)$

\begin{tabular}{lccc}
\hline \hline Component & Mass, kg & $\begin{array}{c}\text { Volume, 1U } \\
\text { fraction }\end{array}$ & $\begin{array}{c}\text { Maximum } \\
\text { power, W }\end{array}$ \\
\hline $\begin{array}{l}\text { CAT thruster } \\
\text { Propellant tank } \\
\text { (minimum size) }\end{array}$ & 0.5 & 0.10 & $3-300$ \\
$\begin{array}{l}\text { Blue Canyon XB1 bus } \\
\text { (GNC, CDH, EPS) }\end{array}$ & 0.25 & 0.10 & -- \\
$\begin{array}{l}\text { Iris transponder } \\
\text { Antennas (telecom) }\end{array}$ & 1.5 & 1 & 2.5 \\
$\begin{array}{l}\text { PPU } \\
\text { Structure/shielding/radiation }\end{array}$ & 0.40 & 0.4 & 12.8 \\
$\begin{array}{l}\text { Deployable solar panels } \\
\text { Total (no extra batteries or }\end{array}$ & 0.10 & 0.05 & -- \\
propellant) & 3.03 & 0.05 & - \\
$\begin{array}{l}\text { Maximum allowable } \\
\text { Allowance for extra batteries }\end{array}$ & 7 & 0.19 & -- \\
and propellant & 3.68 & 1.49 & -- \\
\hline \hline
\end{tabular}

propellant because this is considered later in the orbit-raising analysis. Consistent with the CubeSat design philosophy and to obtain a low mass and cost solution, we have mainly selected commercial off-the-shelf (COTS) components. The CAT thruster fits within the $3 \mathrm{U}$ form factor, as shown in Fig. 1 The total system mass and volume are significantly lower than the mass and volume constraints $(5 \mathrm{~kg}, 3 \mathrm{U}=10 \times 10 \times 34 \mathrm{~cm})$, and there are many feasible vehicle configurations.

The Blue Canyon XB1 bus provides most major CubeSat subsystems including state-of-the-art guidance, navigation, and control system (GNC) systems, command and data handling (CDH), and electric power system (EPS) [32]. The active attitude determination and control system (ADCS) is required to achieve the desired thrust vector throughout maneuvers. The XB1 ADCS consists of a reaction wheel assembly, internal measurement unit, magnetometer, torque rods, sun sensors, star trackers, and GPS. Their system, although currently designed to operate in LEO, is being designed for extended missions in interplanetary locations. Conventional desaturation techniques are not appropriate for interplanetary spacecraft; for example, magnetometers cannot be used for reaction wheel desaturation as conventionally done in LEO environments. At high altitudes beyond LEO, reaction wheel saturation is dominated by solar pressure, and careful control of solar panel orientation can overcome this challenge. The Blue Canyon bus contains a battery with an energy storage of $25 \mathrm{~W} \cdot \mathrm{h}$.

The Iris transponder is selected because it is the lowest mass, volume, and power solution for interplanetary small spacecraft, which enables communication and tracking using the Deep Space Network on X-band frequencies. Existing uhf, S-band, and X-band systems do not currently have the capability to return data at meaningful rates to Earth from distances beyond LEO on small spacecraft form factors. Furthermore, conventional navigation techniques such as using Earth's magnetic field and the GPS constellations are not feasible beyond LEO.

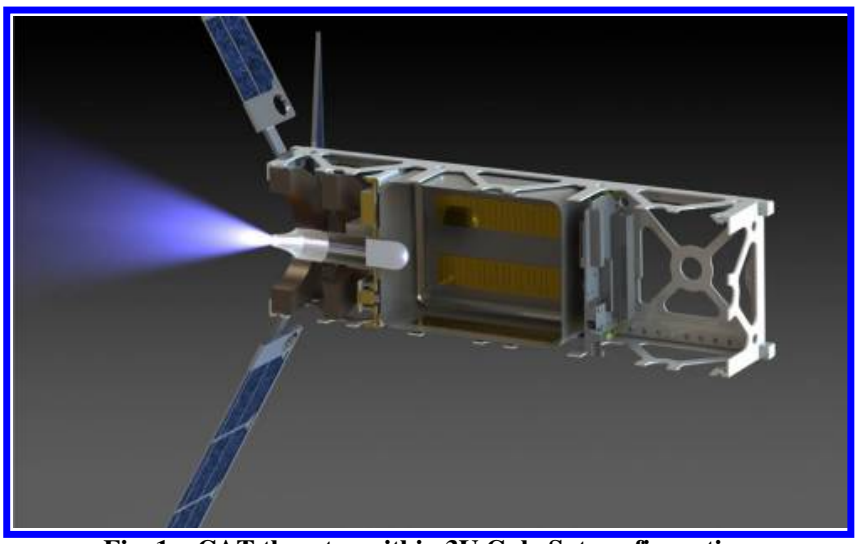

Fig. 1 CAT thruster within 3U CubeSat configuration.
The solar panels are sized to generate approximately $30 \mathrm{~W}$ at 1 astronomical unit (AU), which consists of body-mounted panels on three of the long spacecraft sides and two-sided deployable panels for a total of four $3 \mathrm{U}$ deployed panels. Maximizing the amount of power increases the transfer time and minimizes the required propellant mass. Power collection potential will degrade as the panels age. The master equipment list (MEL) does not include propellant or additional Li-ion 18650 batteries (which each store approximately $15 \mathrm{~W} \cdot \mathrm{h}$ ) because these elements are considered in the trade space.

\section{Model}

A systems-level model is essential to capture all dynamics and constraints of this problem, particularly because CubeSats are highly integrated and have limited available resources. The multidisciplinary integrated model consists of analytic representations of the propulsion system, orbital dynamics, energy collection and management system, battery dynamics, and radiation dosage.

\section{A. Propulsion}

The CAT design focuses on maximizing the thrust-to-power ratio at a specific impulse on the lower end of electric propulsion devices. It achieves a higher thrust-to-power ratio by efficiently ionizing a relatively high flow rate of propellant, about 2-3x higher flow rates than Hall-effect thrusters [33]. The CAT engine fits within a small spacecraft form factor (less than $0.1 \mathrm{U}$, where a $\mathrm{U}$ is $10 \times 10 \times 10 \mathrm{~cm})$. A system block diagram of the CAT propulsion system is given in Fig. 2. Most components of CAT are at TRL 3-4.

A magnetized helicon discharge is used for this highly efficient ionization process without the need for a separate electron source such as a hollow cathode [34]. This system is designed to provide no resultant magnetic dipole. The CAT thruster uses a high plasma density RF helicon source with a converging-diverging throat combined with an expanding magnetic nozzle; see Fig. 3 . A wide variety of propellants are possible due to the electrodeless nature of the helicon plasma source, including propellants stored as liquids or solids. Iodine (nominal propellant), liquid water, ionic fluids, Galinstan, ammonia, butane, alcohols, ethylene glycol, and others are possible options. Iodine propellant is the nominal propellant due to its high storage density and low cost. $I_{\mathrm{sp}}=1010 \mathrm{~s}$ is selected because this maximizes the thrust-to-power ratio; see Fig. 4a.

To obtain a large accelerating electric field (ambipolar acceleration), an efficient helicon RF plasma source is used to generate a very high plasma density $\left(10^{20} \mathrm{~m}^{-3}\right)$ and high electron temperature (20-30 eV) with a variety of propellants (see Fig. 4) and allows the plasma to expand in a magnetic nozzle. Initial optimization has started for a permanent magnet nozzle, which promises to have a nozzle efficiency of greater than $90 \%$. We expect a system efficiency of greater than $50 \%$, significantly exceeding the system efficiency of previous electrospray thrusters, RF double-layer thruster concepts, and miniature ion and Hall-effect thrusters for CubeSats $[35,36]$. A more detailed description of the thruster and justification of these performance parameters is given in [33-37].

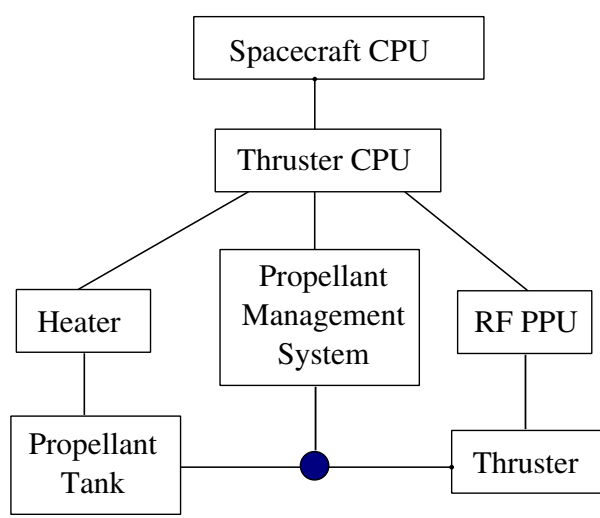

Fig. 2 CAT block diagram. 


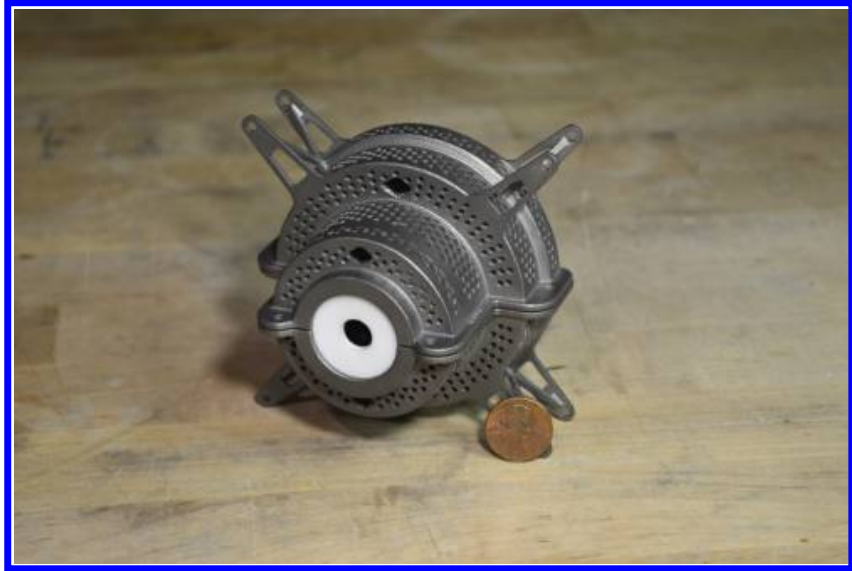

Fig. 3 CAT prototype.

The power processing unit (PPU) is a prototype laboratory unit at an approximate Technology Readiness Level (TRL) of 2/3. Up to $50 \mathrm{~W}$ of power has been produced and applied into a plasma load with a dc to RF efficiency of $93 \%$. The PPU uses high-efficiency MOSFET units for the conversion of dc power at 40 to $50 \mathrm{~V} \cdot \mathrm{dc}$ to RF near the industrial standard of 27.12 MHz. The PPU consists of all solid-state components and is prematched to the plasma load at a single operating set point of choice. Future versions will employ a dynamic matching circuit that adjusts output impedance to match the plasma impedance for a wide throttling range.

\section{B. Orbit}

Two types of orbit-raising strategies orbital dynamics are considered in this section. The first is the conventional constant lowthrust approach based on a simple two-body gravity model, which typically minimizes orbit transfer time. The spacecraft orbit radius from the center of the Earth, $r$, is modeled as

$$
\begin{aligned}
& \dot{r}=2 a \sqrt{\frac{r^{3}}{\mu}} \\
& a=-\frac{\dot{m} V_{\mathrm{ex}}}{m} \\
& V_{\mathrm{ex}}=g I_{\mathrm{sp}}
\end{aligned}
$$

where $a$ is the acceleration, $\mu$ is the Earth's gravitational constant, $\dot{m}$ is the propellant flow rate, $V_{\mathrm{ex}}$ is the exhaust velocity, $m$ is the spacecraft mass, $g$ is the gravitational acceleration, and $I_{\mathrm{sp}}$ is the specific impulse. For CAT, $\dot{m}$ scales linearly with power $p$, where $\delta$ is the mass flow rate to power ratio as in Fig. $4 \mathrm{~d}$ (e.g., $0.1 \mathrm{mg} / \mathrm{s}$ at $10 \mathrm{~W})$ :

$$
\dot{m}=\delta p
$$

The second orbit-boosting approach for orbit raising consists of performing short, high-thrust maneuvers at perigee, which can yield propellant-optimal solutions [38]. This approach may also yield preferable solutions with respect to radiation avoidance because it results in an eccentric orbit that minimizes exposure to the Earth's radiation belts to minimize accumulated radiation dose. In the idealized case, where the thrusts are short and centered at perigee, the increase in apogee is computed using conservation of energy and angular momentum, in Eqs. () $)$ and (י), respectively:
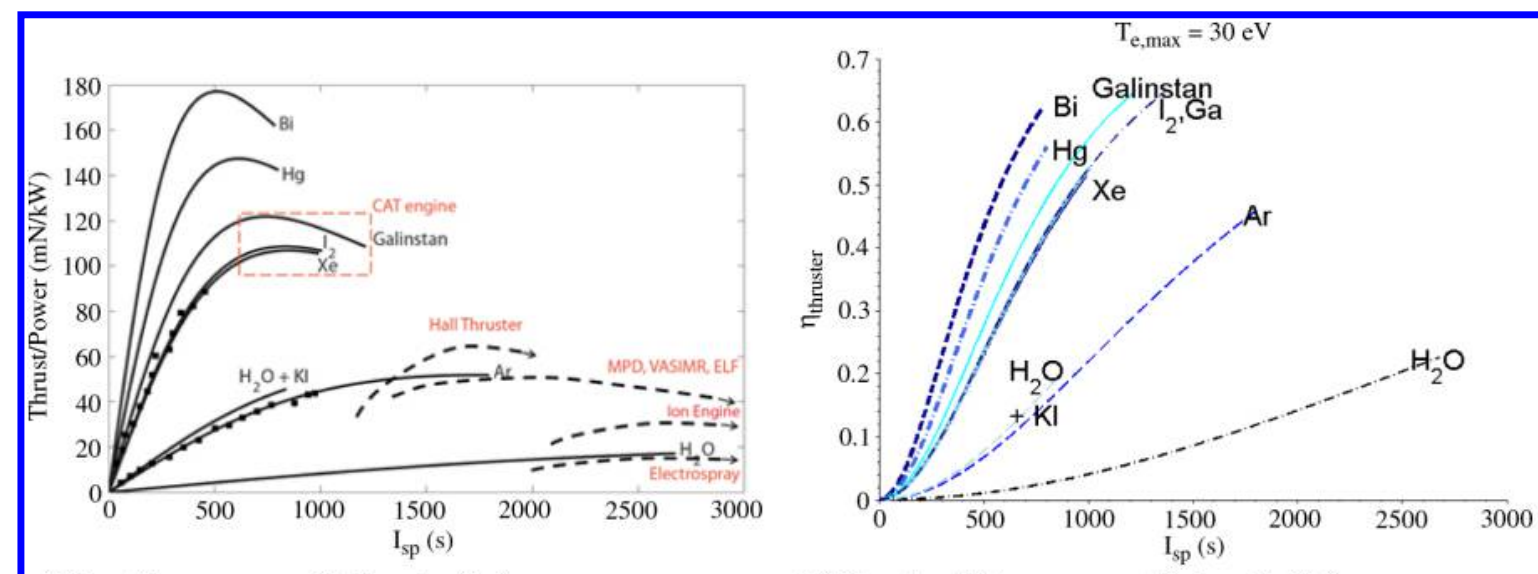

a) Thrust/power vs specific impulse $\left(I_{s p}\right)$

b) Thruster efficiency vs specific impulse $\left(I_{s p}\right)$
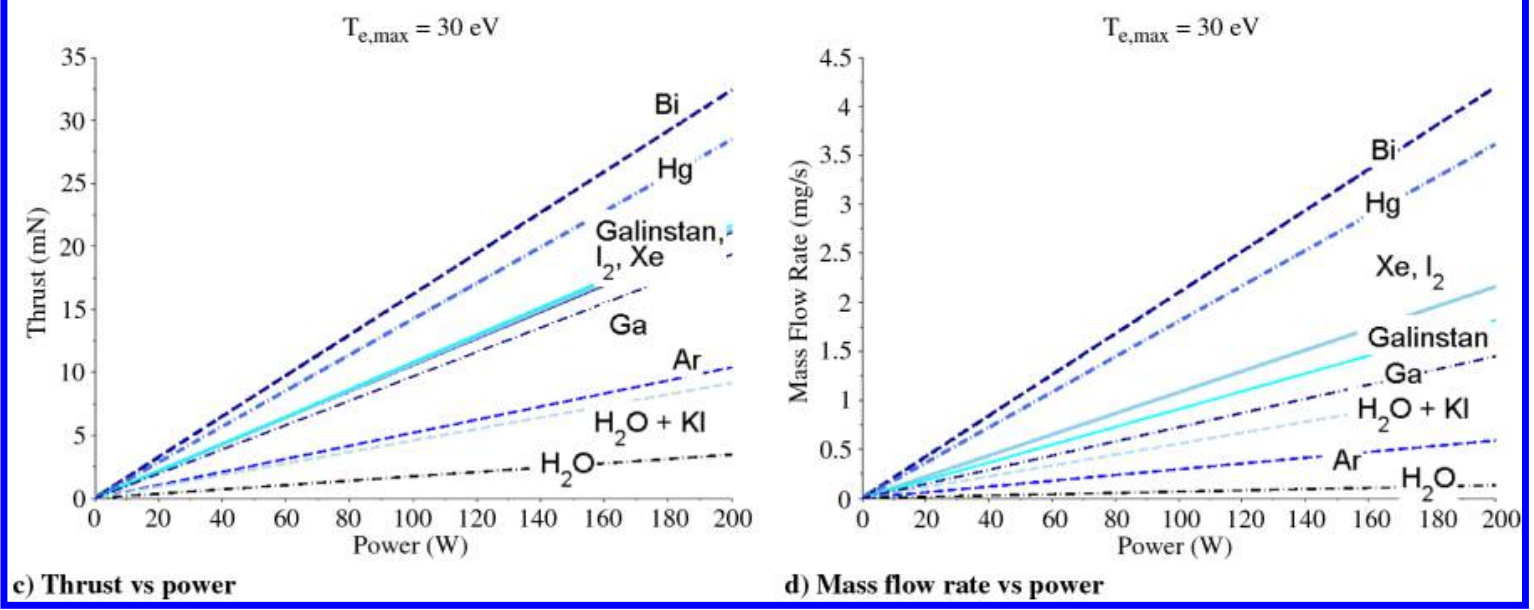

Fig. 4 CAT performance curves for $T_{e, \max }=30 \mathrm{eV}$. 


$$
\begin{gathered}
\frac{v_{p}^{2}}{2}-\frac{\mu}{r_{p}}=\frac{v_{a}^{2}}{2}-\frac{\mu}{r_{a}} \\
r_{p} v_{p}=r_{a} v_{a}
\end{gathered}
$$

where $r_{p}$ and $r_{a}$ are the perigee and apogee radius, $v_{p}$ and $v_{a}$ are the perigee and apogee velocity.

With a $\Delta V$ maneuver, the increase in perigee velocity is $v_{p}^{*}=$ $v_{p}+\Delta V$. By combining Eqs. (5) and (6) to obtain the following quadratic equation:

$$
r_{a}^{* 2}\left(\frac{v_{p}^{* 2}}{2}-\frac{\mu}{r_{p}}\right)+r_{a}^{*} \mu-\frac{\left(v_{p}^{*} r_{p}\right)^{2}}{2}=0
$$

which can be used to solve for the change in apogee altitude $r_{a}^{*}>r_{a}$, and subsequently the apogee velocity $v_{a}^{*}<v_{a}$ using Eq. (6), where $r_{p}$ remains constant throughout each high-powered thrust maneuver.

\section{Energy}

The energy model consists of constraints to ensure feasibility of solutions, which are related to ensuring a positive energy balance and that eclipses and high-power thrust maneuvers can be survived. These constraints are enforced over discrete periods (instead of as a continuous function) to simplify and facilitate optimization of realistic problems.

First, there must be a positive energy balance every orbit, that we collect more energy than is consumed:

$$
p_{s, i} t_{s, i} \geq p_{t, i} t_{t, i}+p_{n, i}\left(t_{s, i}+t_{e, i}\right), \quad \forall i \in I
$$

where $t_{s, i}, t_{e, i}$ are the time in sun and eclipse, and $p_{s, i}$ and $p n, i$ are the average power generated in the sun and consumed by nominal operations, for every orbit $i \in I$. Under the reasonable assumption that thrusting does not occur during eclipse in the thrust-at-perigee approach, the combination of constraints in Eqs. () and (9) is conservative.

For the thrust-at-perigee approach, every thrust maneuver must not deplete the energy stored in the battery:

$$
e_{b} \geq\left(p_{t, i}+p_{n, i}\right) t_{t, i}, \quad \forall i \in I
$$

where $e_{b}$ is the battery capacity, $p_{t, i}$ is the thrust power setting, and $t_{t, i}$ is the thrust duration for every orbit $i \in I$, where there is one maneuver per orbit.

For the constant-thrust case, the spacecraft must be able to sustain eclipses:

$$
e_{b} \geq\left(p_{n, i}+p_{t, i}\right) t_{e, i}, \quad \forall i \in I
$$

\section{Batteries}

Given the strict mass and volume requirements, small spacecraft are using high specific lithium-ion (Li-ion) batteries because they have a high power-to-weight ratio (averaging $200 \mathrm{~W} \cdot \mathrm{h} / \mathrm{kg}$ ) [39]. In the vehicle design, we use conventional $18650 \mathrm{Li}$-ion batteries, which unfortunately experience significant degradation throughout many cycles. For the batteries selected, battery degradation is a function of the cycle number $c$. The battery capacity $e_{b}$ (in kilojoules) is given by the power relationship [40]:

$$
e_{b}=2735.2 e^{(-6 E-4 c)}+64.8
$$

where the maximum of $100 \%$ depth of discharge is assumed on every cycle. If the battery experiences less depth of discharge, less significant battery degradation would be expected; however, there are no available models for this behavior, and so we assume this

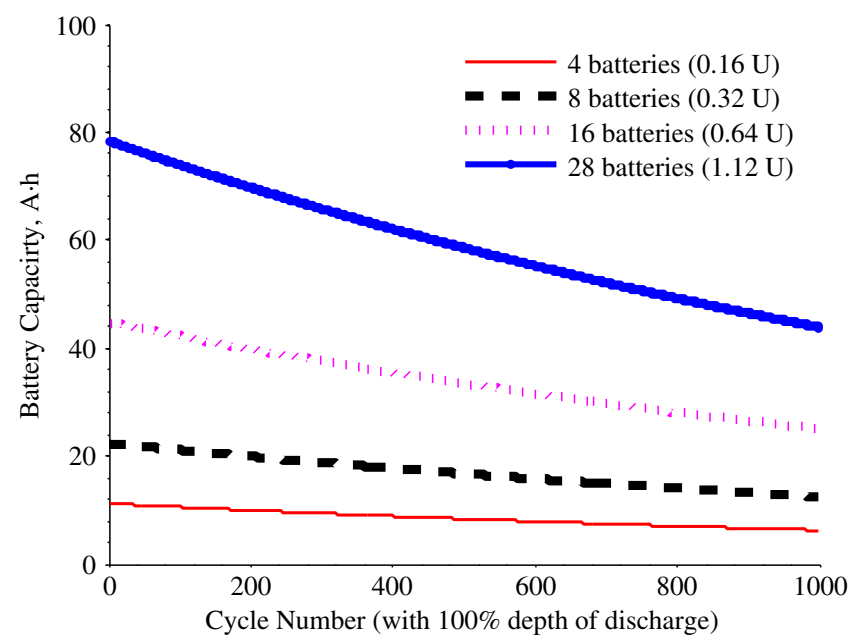

Fig. 5 NCR-18650 battery typical life characteristics.

conservative model. The available capacity is shown in Fig. $\underline{5}$ as a function of the number of 18650 batteries [40]. We assume that 25 batteries can be packed into a $1 \mathrm{U}$ form factor, or each battery takes up about $0.04 \mathrm{U}$.

Ultracapacitors, such as the Ioxus iCAPs [41], which can tolerate $100 \%$ depth of discharge for hundreds of thousands of cycles and provide high power and high charging, were also investigated. The disadvantage of this technology is that ultracapacitors have a significant reduction in energy density $(6 \mathrm{~W} \cdot \mathrm{h} / \mathrm{kg}$ for ultracapcitors vs $200 \mathrm{~W} \cdot \mathrm{h} / \mathrm{kg}$ for lithium-ion batteries). It was established that, for the studies we are investigating, these were not advantageous because of the low initial capacity and the number of cycles we require is in the order of several hundred. If on the other hand, a particular mission requires several thousands of near $100 \%$ discharge cycles, the use of ultracapcitors may be beneficial.

\section{E. Radiation}

Radiation is also a concern for CubeSats, and so we use Analytical Graphics, Incorporated (AGI's) space environment and effects tool module APEXRAD, which provides radiation models based on data collected by the APEX Space Radiation Dosimeter [42]. This model accounts for electron and proton dose and flux as a function of altitude and inclination. The model provides total accumulated radiation dosage based on the trajectories in AGI's Simulation Tool Kit (STK) behind shielding thicknesses of 4.3, 83, 230, and 460 mil of aluminum. The effect of high-energy protons from solar flares, which can contribute to the total radiation dose for interplanetary missions, is accounted for using the Jet Propulsion Laboratory solar proton model from [43].

\section{Constant-Thrust Results}

This section investigates sensitivity analysis relative to key mission parameters and the special case of sun-synchronous orbits, as outlined in Table 2. The baseline orbit transfer case considered is where there is constant thrust in the antivelocity direction from the initial to final orbit, which in general is the time-optimal approach for orbit boosting.

Figure $\underline{6}$ shows orbital velocity as a function of altitude in the Earth system based on a two-body model. This provides a useful worst-case guide on the $\Delta V$ requirements needed to transition between a variety of orbits (e.g., from low LEO, to high LEO, GEO, the moon, or the Earth's SOI) if performing the conventional constant-thrust maneuvers that result in a spiral trajectory.

\section{A. Sensitivity to Mass and Power}

To establish feasibility and quantify the constraints of the constantthrusting approach for a small spacecraft, we consider the case of 
Table 2 Constant-thrust cases studied

\begin{tabular}{lc}
\hline \hline Investigation & Section \\
\hline Sensitivity to mass and power & IV.A \\
Sensitivity to eclipses and $\beta$ angles & IV.B \\
Sensitivity to battery constraints & IV.C \\
Special cases: sun-synchronous orbits & $\underline{\text { IV.D }}$ \\
\hline \hline
\end{tabular}

boosting a 3U CubeSat with CAT from LEO to Earth escape. Spangelo and Longmier [26] demonstrated that it is possible to escape Earth's sphere of influence (SOI) with only $2.5 \mathrm{~kg}$ of propellant and in less than one year (259 days) using $I_{2}$ propellant with an $I_{\mathrm{sp}}=1010 \mathrm{~s}$, and $10 \mathrm{~W}$ of thrust, which is reasonable for a $3 \mathrm{U}$ CubeSat to continually support. The amount of propellant and transfer times to achieve high LEO and GEO orbits are reasonable for a small spacecraft mission. These results indicated that $I_{2}$ is the most time and propellant efficient; thus, it will be used for the remaining analysis.

Sensitivity of a small spacecraft with the CAT thruster to both propellant mass and power levels is shown in Fig. 7. Lower mass systems can achieve significantly higher $\Delta V$ values, as in Fig. 7a, and escape Earth orbit significantly faster, as in Fig. 7b. Emerging deployable solar arrays extend the total power collection area such

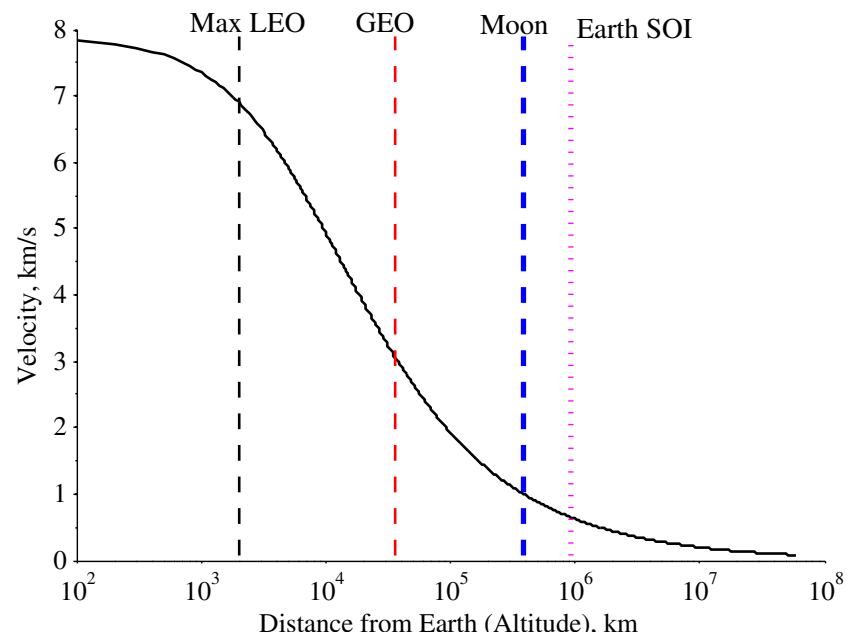

Fig. 6 Orbital velocity as a function of distance from Earth to maximum LEO, GEO, moon orbit, Earth SOI.
Table 3 Solution properties with constant thrusting

\begin{tabular}{lccc}
\hline \hline Power value, W & Propellant mass, kg & Number of orbits & Number of days \\
\hline 10 & 2.5 & 1322 & 269 \\
20 & 2.5 & 681 & 134 \\
25 & 2.5 & 545 & 108 \\
\hline \hline
\end{tabular}

that CubeSats may be able to collect as much as 20 or $30 \mathrm{~W}$ when in the sun. Higher power levels can significantly reduce escape time; see Fig. $7 \mathrm{~b}$ and Table 3 . The time to achieve a given orbit boost or change in $\Delta \bar{V}$ is a power-law function of the power level because the time is proportional to the inverse of mass flow rate, which is proportional to the power level; see Eq. (4). The escape time is independent of propellant mass (i.e., all cases in Fig. $7 \mathrm{~b}$ require the same amount of propellant mass). Furthermore, because higher-powered systems escape Earth orbit quickly, they also have significantly lower total accumulated radiation dose in the first year.

\section{B. Energy and Eclipse Constraints}

The ability to continually sustain a certain thrust value is limited by the duration of eclipses; see Eqs. () and (9). Typically, CubeSats are initially placed into high-latitude LEOs that have eclipses on the order of one-third the orbit [30]. Worst-case eclipse fraction of orbit and total eclipse time are shown in Fig. 8 for different circular orbits as a function of latitude. The results shown are worst-case because they assume that the line of sight to the sun lies in the orbit plane, where the angle between the spacecraft orbit plane and the line of sight to the sun $\beta=0 \mathrm{deg}$, and so maximum eclipse is always experienced. Eclipse fractions vary throughout the year due to orbital precession; see [30].

The eclipse duration (Fig. $\underline{\text { c }}$ ) is a product of the orbital period and eclipse fraction, which each vary as a function of altitude differently. The orbital period is given by $P=2 \pi \sqrt{r^{3} / \mu}$, where $P$ is the orbital period and $r$ is the orbit radius ( $r=a+R_{E}$ ), where $a$ is altitude and $R_{E}$ is the Earth's radius; $\mu$ is the gravitational constant in Fig. 9a. The orbital period is monotonically increasing, but as a function of $\sqrt{r^{3}}$. The eclipse fraction is computed based on the orbit geometry and is a function of $\sin ^{-1}(1 / r)$, as in Fig. $8 \mathrm{~b}$. Thus, it decreases monotonically with increasing $r$, but at a faster rate for lower $r$ values. The eclipse durations decrease slightly as the altitude increases from the lower to higher range of LEOs $(300-1000 \mathrm{~km})$ in Fig. $8 \mathrm{c}$ because the eclipse fraction decreases at a relatively higher rate than the orbital period increases; see Fig. 8d. For altitudes between 1000 and $100,000 \mathrm{~km}$, eclipse durations increase because the eclipse fraction decreases at a lower rate relative to the increase in orbital period, and for altitudes exceeding $100,000 \mathrm{~km}$, the eclipse durations approach zero, following the eclipse fraction trends.

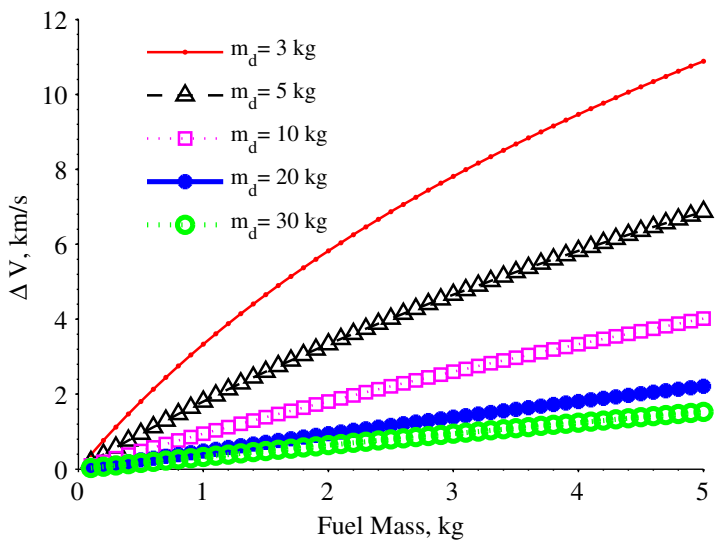

a) $\Delta V$ as a function of propellant mass, where $m_{d}$ is spacecraft dry mass

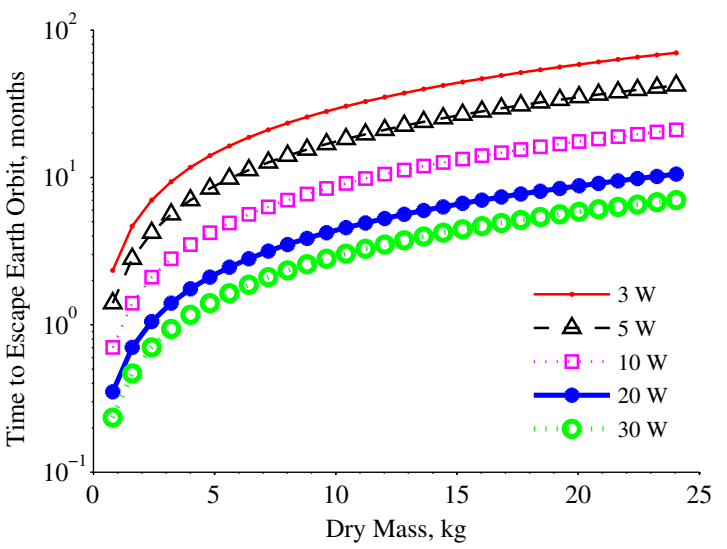

b) Earth escape time for initial $500 \mathrm{~km}$ circular orbits (requires $\Delta V=6.96 \mathrm{~km} / \mathrm{s}$ ) with different power levels

Fig. 7 Sensitivity of CAT thruster to propellant mass and power level assuming $I_{2}$ propellant and $I_{\mathrm{sp}}=1010 \mathrm{~s}$. 


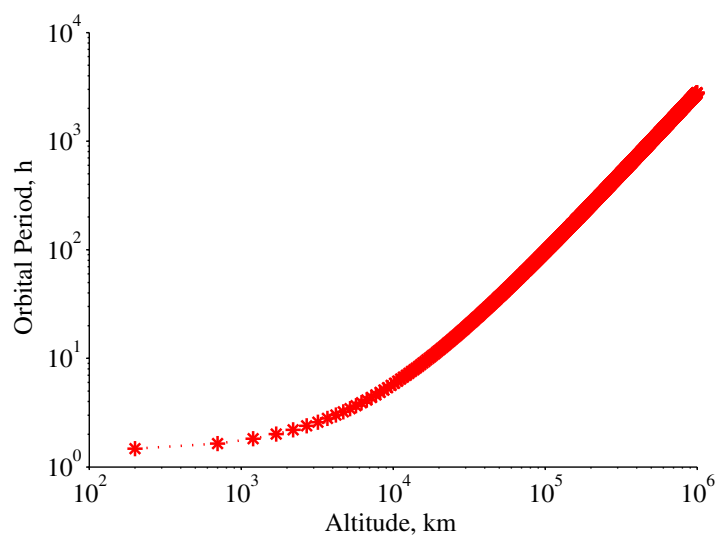

a) Orbit period

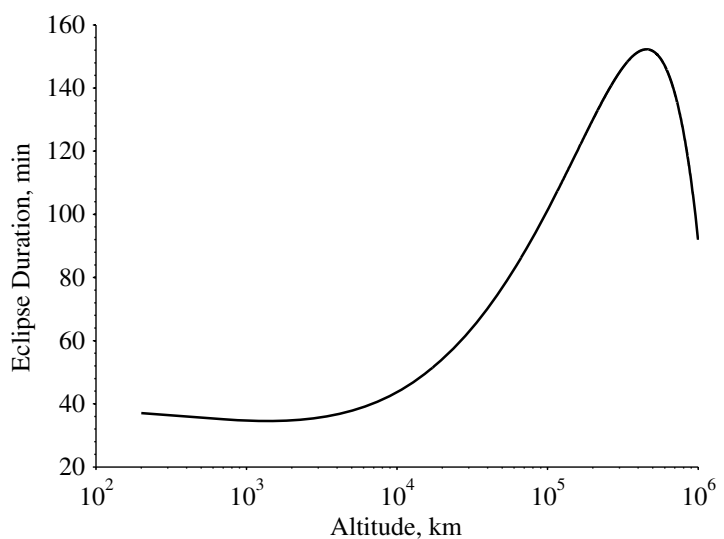

c) Total time spacecraft is in eclipse every orbit

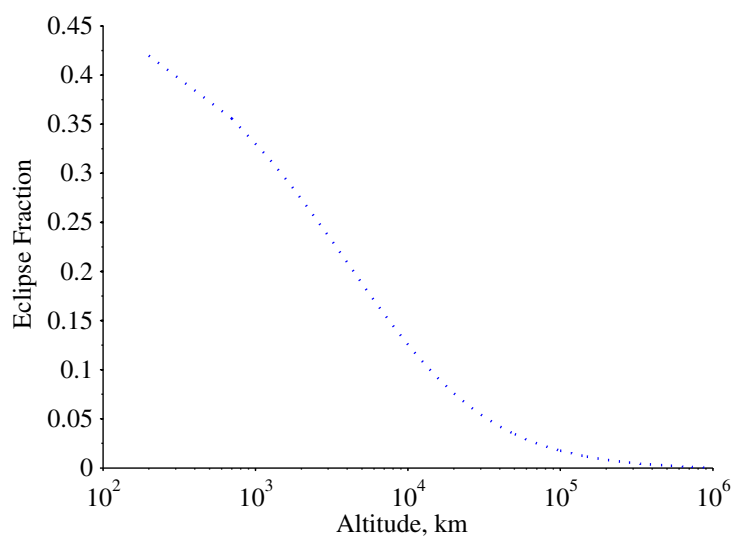

b) Fraction of orbit spacecraft is in eclipse

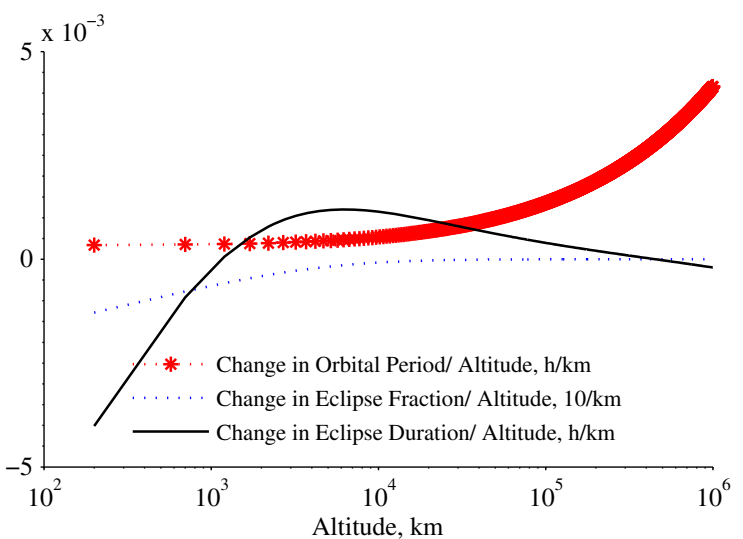

d) Rates of change of eclipse properties

Fig. 8 Worst-case $(\beta=0$ deg) eclipse properties for circular polar LEOs.

Figure 8 showed the worst-case eclipse durations, but both eclipse duration and time to achieve an altitude with no eclipses depends on $\beta$ and power setting; see Fig. 10a. In the worst case condition, where $\beta=0 \mathrm{deg}$, as in Fig. 8 , this altitude is greater than $100,000 \mathrm{~km}$; however, the altitude where eclipses are no longer experienced can vary several orders of magnitude with different $\beta$ and power values.

\section{Impact of Battery Constraints}

To minimize the time to transfer an orbit from a $500 \mathrm{~km}$ LEO to escape Earth orbit with the constant-thrust approach, the optimal strategy is to use the maximum feasible power satisfying Eqs. (8) and (9) throughout the mission. The limiting factor may evolve throughout the mission, as eclipse durations and fractions change, and are a function of the battery capacity and nominal energy consumption, $E_{\text {batt }}$ and $P_{n, i}$.

To quantify the impact of battery and energy balance constraints, we consider worst-case orbit from an eclipse perspective $(\beta=0 \mathrm{deg})$ with two different battery sizes: one with the conventional $25 \mathrm{~W} \cdot \mathrm{h}$ battery (included in the Blue Canyon bus in Table 1), and one with four additional $15 \mathrm{~W} \cdot \mathrm{h}$ Li-ion 18650 batteries (each weight $0.05 \mathrm{~kg}$ ); see Fig. 11 . For the $25 \mathrm{~W} \cdot \mathrm{h}$ battery case, the beginning of the mission is constrained by the energy balance [Eq. (8)], whereas the later part of the mission (after 0.3 years) is constrained by the battery capacity [Eq. (9)]; see Fig. 11a. For the higher battery capacity case, the entire mission is constrained by the energy balance; see Fig. $11 \mathrm{~b}$. The $25 \mathrm{~W} \cdot \mathrm{h}$ case requires 0.51 years to escape Earth orbit, with an initial mass of $6 \mathrm{~kg}$, yielding a final mass of $3 \mathrm{~kg}$. On the other hand, the $85 \mathrm{~W} \cdot \mathrm{h}$ case requires 0.45 years to escape Earth orbit. This spacecraft requires an initial mass of $6.4 \mathrm{~kg}$ to yield a final mass of $3.2 \mathrm{~kg}$ to account for the four additional batteries. Thus, although increased battery capacity can reduce constraints and improve performance, because additional mass is required, it may not increase the performance dramatically, as discussed in more detail in Sec. VI in the context of a more sophisticated orbit-boosting approach.

\section{Special Case: Sun-Synchronous Initial Orbits}

In the special case that the spacecraft is launched into a sunsynchronous orbit, it will have no eclipses or shorter initial eclipse times; see Fig. 12a. This reduces or eliminates the need for significant onboard battery storage, and as a result, a high constant-thrust power level can be continually maintained, satisfying Eqs. (ㅁ) and (9). Furthermore, if the initial orbit does experience eclipses (for cases where the initial $\beta<68 \mathrm{deg}$ ), it will achieve an altitude where no eclipses are experienced faster than non sun-synchronous orbits, as in Fig. 10.

LEO sun-synchronous orbits are not always in the sun because $\beta$ varies throughout the year; see Fig. $12 \mathrm{~b}$. Thus, depending on the time of year the spacecraft begins its maneuver, there will be an associated required altitude gain (see Fig. 10) to no longer experience eclipses. In the worst-case sun-synchronous case $(\beta=60 \mathrm{deg})$, only 31 days are required with the $10 \mathrm{~W}$ power case to achieve the required altitude $(1000 \mathrm{~km})$ for no eclipses. The $25 \mathrm{~W}$ case in Fig. 4 is representative of a sun-synchronous terminator orbit $(\beta=9 \overline{0} \mathrm{deg})$, where the available solar power can immediately be provided to the thruster (where it is assumed approximately $5 \mathrm{~W}$ is provided to the spacecraft bus and transponder). In this case, the spacecraft can escape Earth orbit starting from a $500 \mathrm{~km}$ LEO in only 108 days; see Table $\underline{3}$.

\section{Optimal Thrusting at Perigee with Variable Magnitude and Time}

It is possible to reduce the $\Delta V$ requirements shown in Fig. 6 for orbit boosting by performing high-thrust maneuvers near the orbit perigee. This approach increases the perigee speed and apogee altitude, by conservation of momentum and energy, as in Eqs. () and 


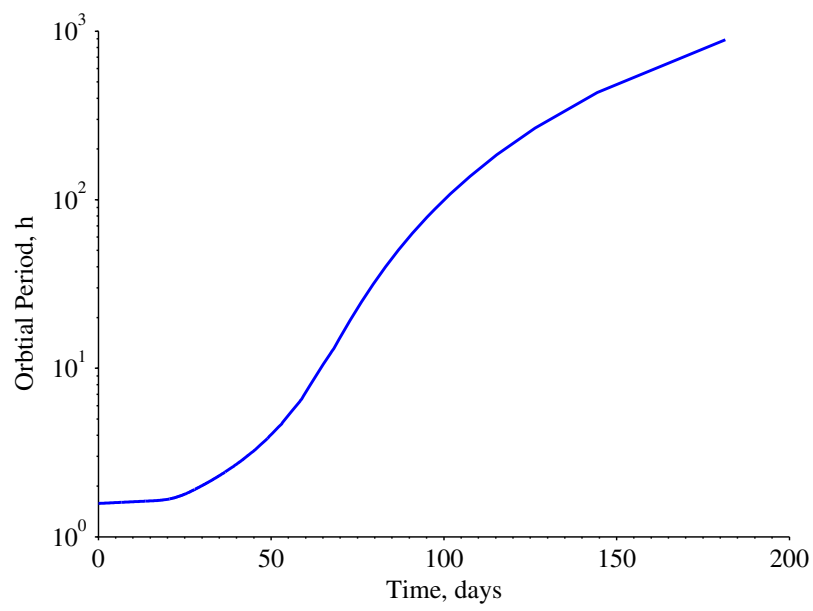

a) Orbital period

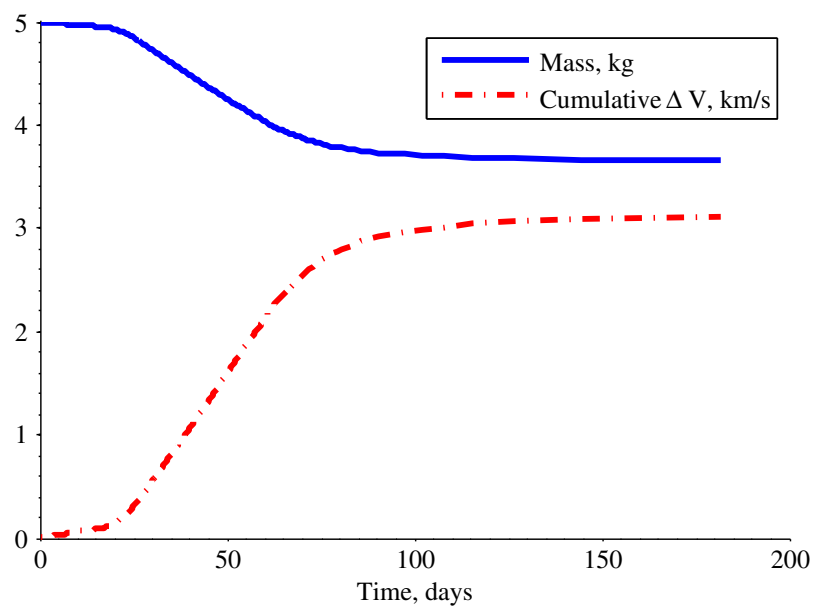

b) Mass and $\Delta V$

Fig. 9 Properties of solutions with 28 batteries, requiring $1.12 \mathrm{U}$ of volume. This approach requires 181 days to escape Earth orbit and $1.35 \mathrm{~kg}$ of $I_{2}$ propellant.

(6) and exploiting the Oberth effect [38]. The thrust-at-perigee approach investigated in [28] can provide significant propellant savings relative to a constant-thrust approach. However, assigning a constant thrust level and duration for every orbit will likely yield suboptimal solutions because it does not allow the trajectory to benefit from the additional energy that becomes available as the spacecraft spirals from Earth and has shorter relative eclipse times. In addition, there may be more efficient times in the mission for higherpowered or longer duration thrusts due to changes in system mass, orbit velocity, and battery capacity (as it degrades throughout the mission with greater charge/discharge cycles). The variable magnitude and time thrusting at perigee approach uses dynamic thrusts to improve in propellant and/or time efficiency in orbitboosting maneuvers capturing the time-dependent variables, dynamics, and constraints. [27]. We formulate this optimization problem in Sec. V.A and solve it in Sec. $\underline{\text { V.B. }}$.

\section{A. Optimization Formulation}

An analytic formulation to optimize the approach for dynamic perigee thrusting for orbit boosting is presented, where the objective function is to minimize the time required to achieve a change in orbit (i.e., LEO to GEO, LEO to Earth escape). The formulation includes analytic system-level dynamics and constraints for the spacecraft vehicle, energy, orbit, propulsion, and battery degradation. The variable thrust and duration optimization formulation goal, decisions, constraints, dynamics, and inputs are given later.

The objective is to find the trajectory that minimizes time to escape Earths sphere of influence (radius: $925,000 \mathrm{~km}$ ) by thrusting for a short time once per orbit, centered at perigee. The objective function is given by Eq. (12), where $T_{\text {escape }}$ is the time require to escape Earth orbit, as in Eq. ( $\underline{13})$ :

$$
\begin{gathered}
\min T_{\text {escape }} \\
T_{\text {escape }}=\sum_{1}^{i^{*}}\left(t_{s, i}+t_{e, i}\right)
\end{gathered}
$$

where $i^{*}$ is the first interval where $r_{i} \geq 925,000 \mathrm{~km}$, where $r_{i}$ is the radius at the end of interval $i$.

The decision variables are the level and duration of the thrust, centered at perigee, where one thrust maneuver is assumed to occur every orbital period. Energy constraints [Eqs. (ㅁ) and (9)] are enforced to ensure that there is a positive energy balance every orbit, and the battery capacity, which varies dynamically as the battery degrades with additional battery cycling, is sufficient to support the thrust maneuvers and eclipses, which also vary dynamically as the orbit changes. The system dynamics are modeled using the propulsion, orbit, and energy models from Sec. IV. There are two decision variables per orbit: what thrust value to use and how long the thrust should last. Realistic CubeSat vehicle volume and mass constraints are also enforced (assuming a $3 \mathrm{U}$ CubeSat), which must account for additional batteries to supplement a nominal onboard battery, the thruster, propellant tank, and propellant, as in Table 1.

The continuous-time problem is discretized into orbit-long intervals, where the dynamics and constraints are enforced over every orbit. The problem is coded and solved in MATLAB using the optimization solver fmincon, a solver for constrained multivariable

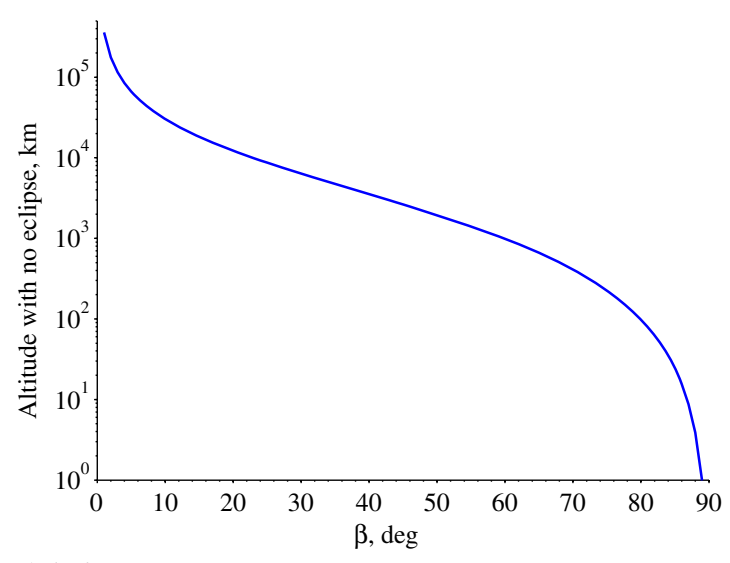

a) Altitude

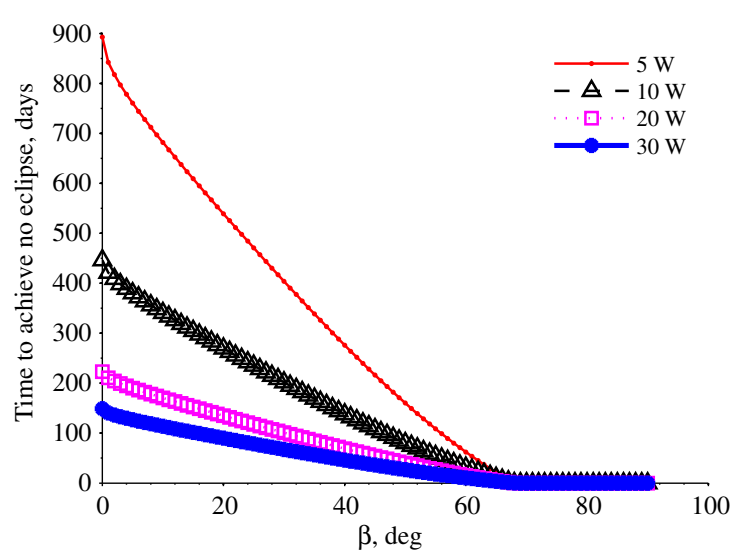

b) Time

Fig. 10 Required altitude and time where there is no longer eclipses as a function of $\beta$, assuming a total initial spacecraft mass of $5 \mathrm{~kg}$ and $I_{2}$ propellant. 


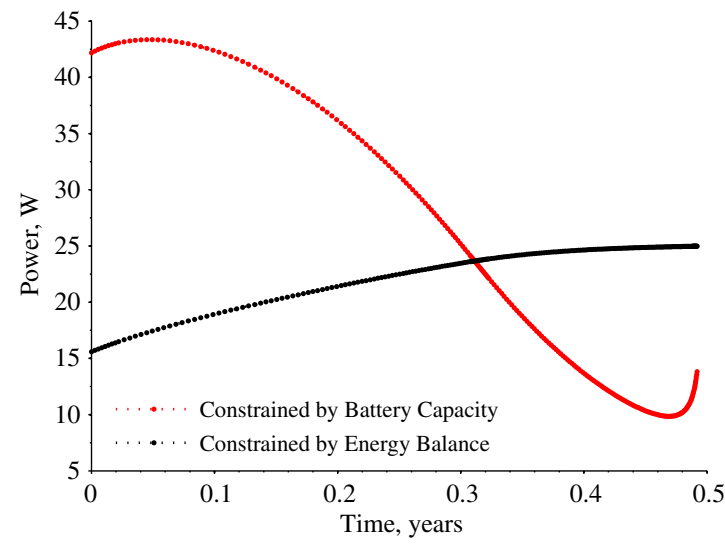

a) $25 \mathrm{Whr}$ battery capacity

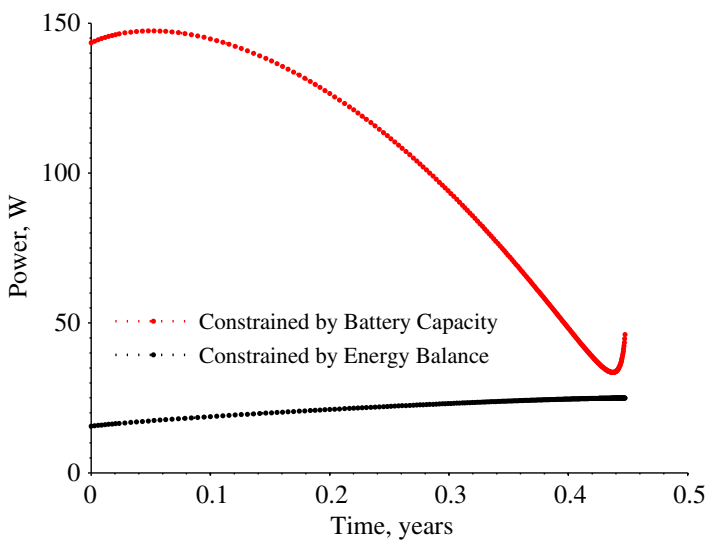

b) $85 \mathrm{Whr}$ battery capacity

Fig. 11 Optimal solutions with realistic power variation.

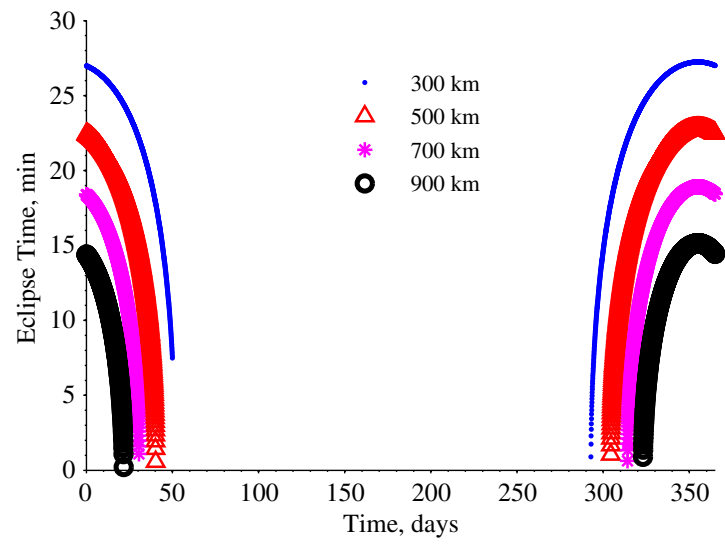

a) Eclipse trends

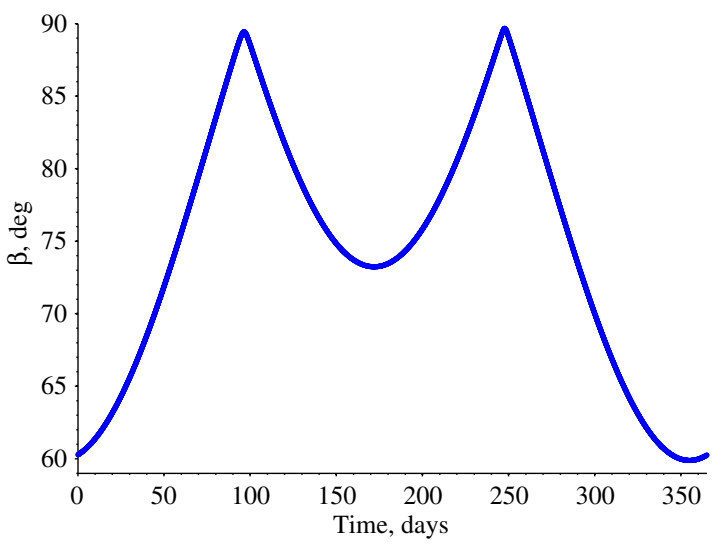

b) Solar $\beta$ angle

Fig. 12 Eclipse trends and $\beta$ (the angle between the spacecraft orbit plane and the line of sight to the sun) trends throughout year for a nearly terminator sun-synchronous orbit.

functions. We optimize the time to transfer a spacecraft from an initial $500 \mathrm{~km}$ circular (non-sun-synchronous) orbit to GEO using the CAT thruster. The thruster power is constrained to between 3 and $300 \mathrm{~W}$; the spacecraft is assumed to nominally collect $30 \mathrm{~W}$ of solar power and consume $3 \mathrm{~W}$ for bus operations.

\section{B. Optimal Solutions}

Optimal solutions assuming different battery volumes for a worstcase non-sun-synchronous orbit ( $\beta=0 \mathrm{deg})$ and a sun-synchronous orbit $(\beta=90 \mathrm{deg})$ are shown in Fig. 13. The relative time advantages with larger batteries are significant, particularly for battery volumes less than $1 \mathrm{U}(1 \mathrm{U} \approx 25$ batteries). The escape time is most dependent on battery capacity and not dependent on whether the orbit is sun-synchronous, and so the energy capacity constraint [Eq. (9)] is active more than the energy balance constraint [Eq. (8)].

Properties of the non-sun-synchronous optimal solutions for different battery sizes are shown in Fig. 14, where the maximum power $(300 \mathrm{~W})$ is used for most of the trajectory; therefore, thrust duration is the true real control in the optimization problem (which explains why Fig. $15 \mathrm{~b}$ is nearly a scaled-up version of Fig. 15a). For all cases, there is an increase in thrust duration and total energy for the first 300 orbits because the fraction of time the spacecraft is in eclipse shrinks as the orbit raising occurs, as shown in Fig. $\underline{8}$. The increase in perigee velocity and apogee altitude are significant at the start of the mission and then grow at reduced levels later in the mission.

All solutions shown in Fig. 13 require $1.35 \mathrm{~kg}$ of $I_{2}$ propellant, which yields a volume of $0.27 \mathrm{U}$. The solution with $1.12 \mathrm{U}$ equivalent (28 batteries) provides the lowest escape time with the most realistic battery volume, yielding a total volume (with propellant and batteries) of $2.88 \mathrm{U}$ and $5.78 \mathrm{~kg}$ (using the parameters in Table 1), which just satisfies the volume constraint (3U). This solution with 28 batteries requires 701 battery cycles and 181 days to achieve the goal of escaping Earth's SOI at an altitude of 925,000 km. Properties of this solution are shown in Fig. 16. The orbit altitude increases exponentially for about the first $10 \overline{0}$ days and then linearly thereafter (see Fig. 16a), resulting in the perigee velocity trend (see Fig. 16b) and orbital period (see Fig. 9a). Propellant mass is also consumed at a faster rate early in the mission, and consumption plateaus later on; see Fig. 9 b.

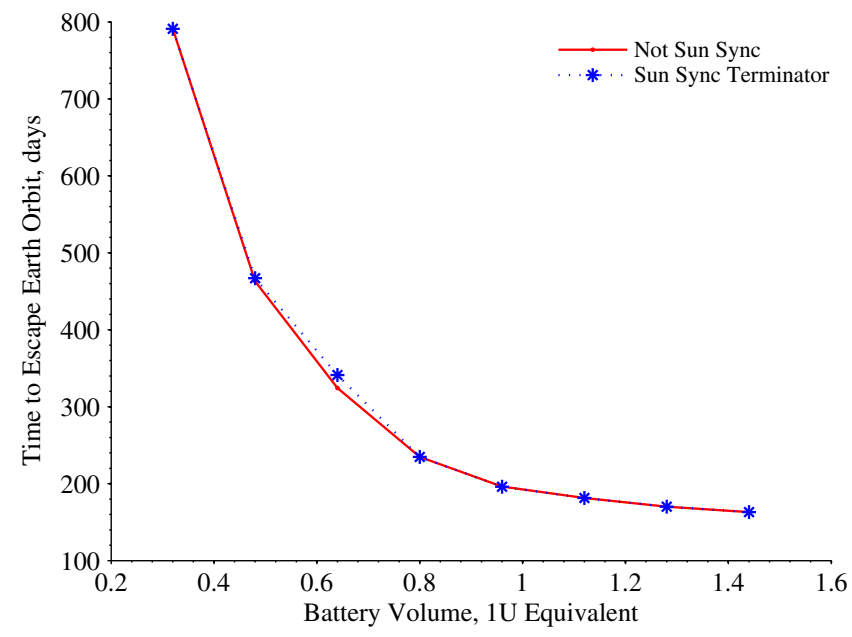

Fig. 13 Optimal variable thrust and duration solutions. 


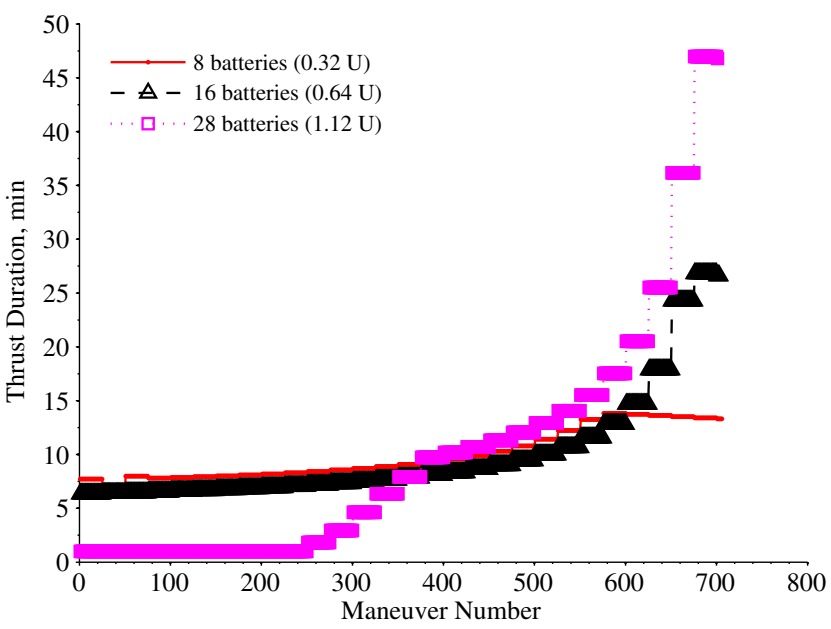

a) Thrust duration vs maneuver number

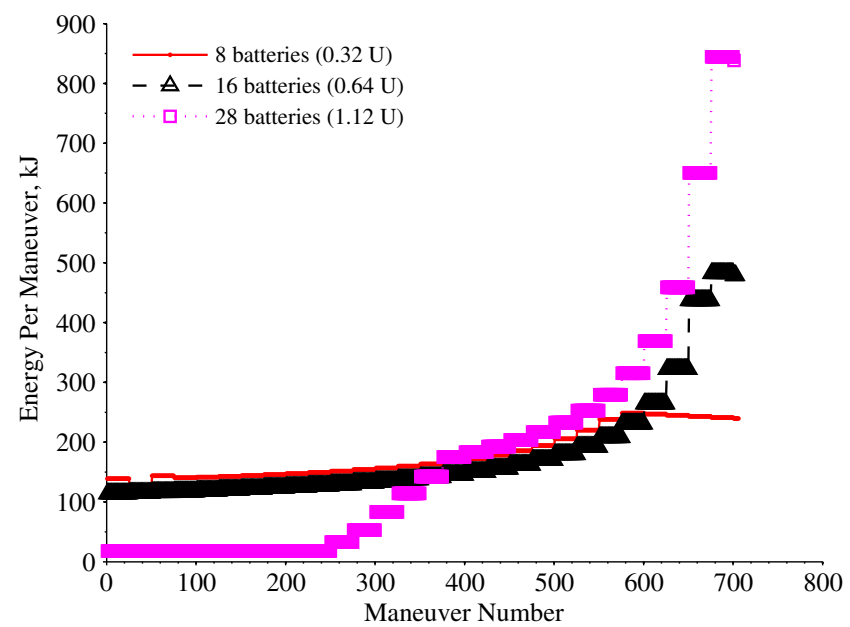

b) Thrust energy vs maneuver number

Fig. 14 Properties of non-sun-synchronous optimal solutions with different battery sizes.

The maximum available energy to satisfy Eqs. (8) and (9) is not used on every orbit; however, the battery degradation is modeled as in Eq. (11), and so the results are pessimistic. The solutions were validated in AGI's STK, and the escape time and propellant consumption results were within a few percent of the results in this section, validating the approach.

\section{Design Considerations}

The performance metrics for small spacecraft systems differ in some cases from conventional metrics for larger spacecraft, such as transfer time and propulsion mass. Small spacecraft are very tightly constrained, and so the required power and/or voltage level, thrust duration, volume, battery cycles, and even total radiation dosage experienced are also important considerations in the design space. Table 4 provides a summary of the key performance metrics for the cases investigated in this paper.

The best approach depends considerably on the goals and constraints of the mission considered. If the goal is to minimize transfer time, the best maneuver approach depends significantly on the power level that can be maintained. If the CubeSat is launched into an initial sun-synchronous orbit and can maintain $25 \mathrm{~W}$ continuously, the continuous thrusting approach will minimize time, but if not, the optimal variable thrust is optimal. If a propellantoptimal solution is desired, the best approach is the optimal variable thrust. However, this approach requires additional batteries, resulting in more volume and mass in addition to the base spacecraft. The

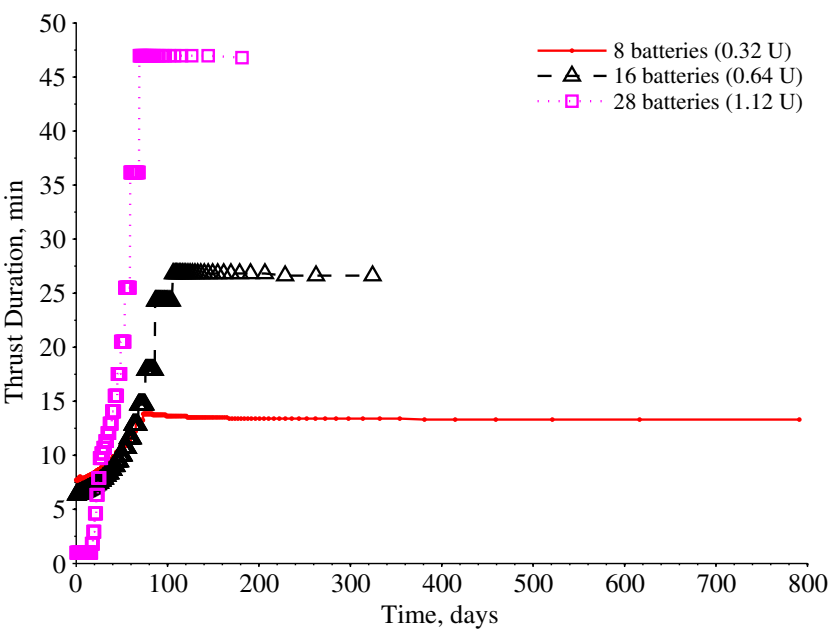

a) Thrust duration vs time

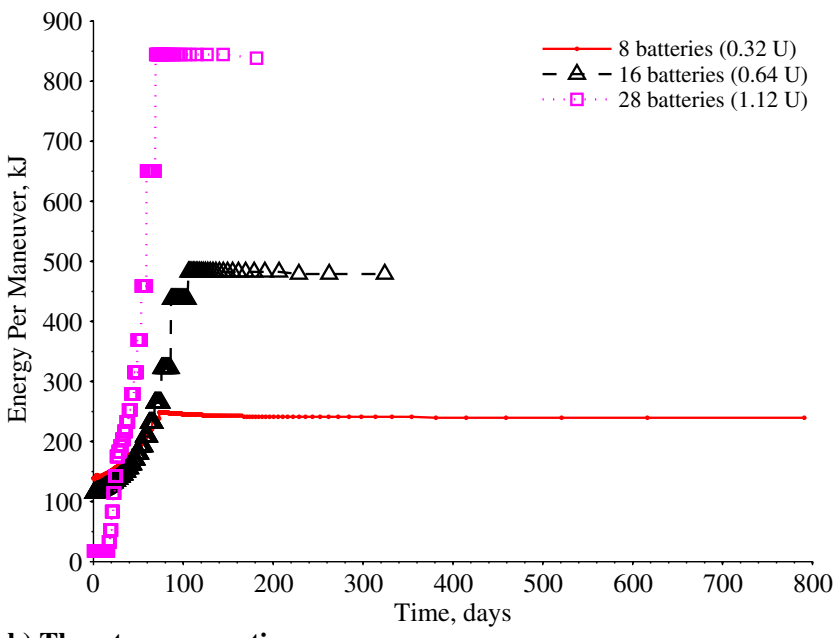

b) Thrust energy vs time

Fig. 15 Properties of non-sun-synchronous optimal solutions with different battery sizes.

optimal variable-thrust option also requires high voltage and power loads and the resulting thermal concerns and significant battery cycling associated with high-powered bursts. On the other hand, the continuous-thrusting approach requires that the thruster is on continuously, which may be operationally challenging to maintain. For example, maintaining constant attitude control may be challenging; the spacecraft will have limited opportunities to mitigate solar pressure by controlling its attitude, dump reaction wheel momentum, and communicate with Earth ground stations (required for tracking and communication).

Attitude control is required to perform the trajectory maneuvers described in this paper. We performed a simple sensitivity analysis for the constant-thrust spiral out trajectories presented in Sec. V relative to angular error (defined at the angle between the ideal and actual thrust vector). We found that the resulting time to reach Earth escape was increased by $0.02,1.5$, and $6.4 \%$ for angular errors of 1,10 , and $20 \mathrm{deg}$, respectively. The impact on time is independent of thrust power level. We expect the attitude errors to have a more significant impact on escape time for the thrusting at perigee approach. Pointing errors can also impact the orbit shape and precession and change. However, the attitude control system is expected to have pointing accuracy less than a tenth of a degree $(0.002 \mathrm{deg}, 1-\sigma)$ [32], and so we expect very marginal impacts on the escape time performance in both cases.

Radiation effects are also a design concern for CubeSats, particularly because they fly COTS components that are not radiation-tolerant, which are flown because of cost, mass, and volume constraints. The solar proton dosage is $1.1 \mathrm{krad}$ for each case at $1 \mathrm{AU}$, and the difference between the cases is due to different 


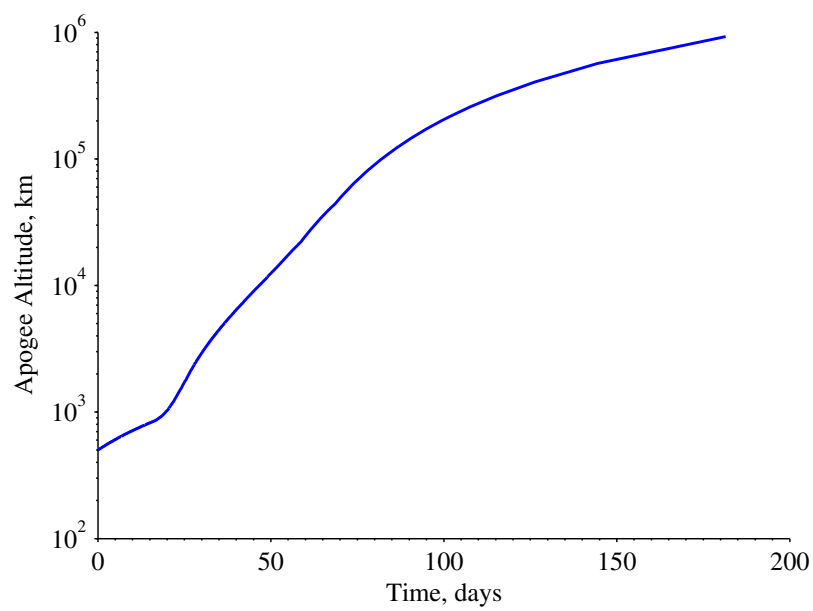

a) Apogee altitude

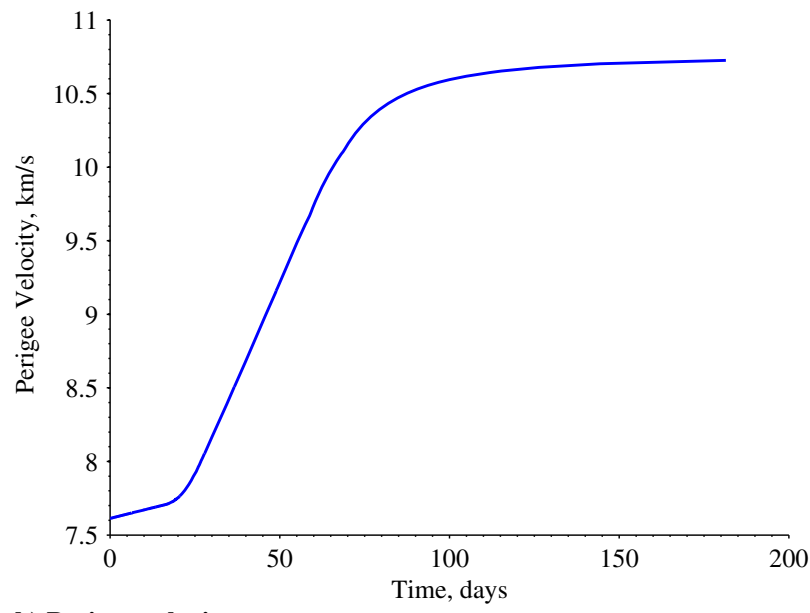

b) Perigee velocity

Fig. 16 Properties of solutions with 28 batteries, requiring $1.12 \mathrm{U}$ of volume. This approach requires 181 days to escape Earth orbit and $1.35 \mathrm{~kg}$ of $I_{2}$ propellant.

electron and proton dosage and flux values [44]. The constant-thrust approaches experience significantly more radiation, whereas the thrust-at-perigee approach results in an elliptical orbit where a large fraction of the orbit is spent out of the geocoronal after the orbit has been boosted (particularly beyond 10 Earth radii). Studies have suggested that select CubeSats components can tolerate between 5 and $20 \mathrm{krad}$ before failing, depending on the amount of shielding [45]. However, modern electronics tend to work better and longer

Table 4 Comparison of maneuver approaches to achieve Earth escape

\begin{tabular}{lcc}
\hline \hline & $\begin{array}{c}\text { Continuous } \\
\text { thrusting in } \\
\text { velocity direction }\end{array}$ & $\begin{array}{c}\text { Optimal variable } \\
\text { thrust magnitude } \\
\text { and duration } \\
\text { at perigee }\end{array}$ \\
\hline Thrust power level & $\begin{array}{c}10 \mathrm{~W} / 25 \mathrm{~W} \\
\text { (sun-synchronous }) \\
\text { Continuous }\end{array}$ & $\begin{array}{c}\text { Variable } \\
(50-300 \mathrm{~W}) \\
1-60 \mathrm{~min}\end{array}$ \\
$\begin{array}{l}\text { Thrust duration } \\
\text { Propellant required for Earth }\end{array}$ & 2.5 & 1.35 \\
$\begin{array}{l}\text { escape, kg } \\
\text { Propellant and battery mass } \\
\text { and volume }\end{array}$ & $2.5 \mathrm{~kg}, 0.5 \mathrm{U}$ & $2.84 \mathrm{~kg}, 1.4 \mathrm{U}$ \\
$\begin{array}{l}\text { Time required for Earth } \\
\text { escape, days }\end{array}$ & $269 / 108$ & 181 \\
$\begin{array}{l}\text { Number of orbits } \\
\text { Total accumulated ionizing } \\
\text { dose with } 232.5 \text { mils (5.9 mm } \\
\text { of Al) after one year, krad }\end{array}$ & $1322 / 545$ & 701 \\
\hline \hline
\end{tabular}

than anticipated in orbit; for example, the University of Tokyo's CubeSat XI-IV survived over a decade of successful operations in a $820 \mathrm{~km}$ sun-synchronous orbit (launched in June 2003) [16]. Radiation effects can mainly be mitigated using latchup-immune parts with linear energy transfer greater than approximately $35-70 \mathrm{MeV} /\left(\mathrm{mg} * \mathrm{~cm}^{2}\right)$, robust software, and periodic power cycling of the system [46]. Generally simple shielding strategies, such as encasing sensitive electronics in aluminum are sufficient to protect against total ionizing dosages [27].

\section{Conclusions}

This paper has developed a modeling framework and simulation environment for evaluating orbit transfers from LEO to Earth escape trajectories and is applicable to any initial and final orbits within the Earth system. We have used these tools to demonstrate the feasibility for a small spacecraft form factor (in particular a $3 \mathrm{U}$ CubeSat) to perform orbit transfers using the CAT thruster technology. Systemslevel constraints such as availability of power, energy, mass, and volume, which limit these small, highly integrated spacecraft, have been considered. The key constraints and sensitivities of using small spacecraft for interplanetary orbit transfers have been identified and quantified. The modeling and optimization approach presented in this paper is independent of the specific form factor, thruster, or any other technology and could be applied to a variety of space systems.

The main contribution of this paper is the demonstration of feasible vehicle and trajectory designs to perform orbit transfers from LEO to Earth escape using small spacecraft as well as the identification of key constraints and tradeoffs. For a small $3 \mathrm{U}$ CubeSat, the constant-thrust approach is the time-optimal solution if the initial orbit is sunsynchronous, whereas the thrust-at-perigee approach is faster if the initial orbit is not sun-synchronous. Although the thrust-at-perigee approach minimizes the required propellant requiring almost half as much as the constant-thrust approach, it will always require more battery capacity and thus more mass and volume. The thrust-atperigee approach can minimize radiation exposure due to its eccentric orbit that avoids long durations exposed to the Earth's radiation belts. The general approach presented in this paper is applicable to a broad case of Earth-orbit or interplanetary mission scenarios where a spacecraft is in any initial orbit about a planet or moon with the goal of escape the body's SOI. For example, if the 3U CubeSat described in this paper starts in GEO or GTO (where significantly lower $\Delta V$ is required to escape Earth orbit), it would escape in a fraction of the time, with less required power, or could accommodate a more massive spacecraft.

\section{Acknowledgments}

The authors acknowledge support by NASA cooperative agreement NNX13AR18A. Part of the research was carried out at the Jet Propulsion Laboratory, California Institute of Technology, under a contract with NASA.

\section{References}

[1] Hofer, R., Mueller, J., and Ziemer, J., "Survey of Propulsion Technologies Applicable to Cubesats," Proceedings of the 57th Joint Army-Navy-NASA-Air Force (JANNAF) Propulsion Meeting, Colorado Springs, CO, 2010.

[2] Selva, D., and Krejci, D., "A Survey and Assessment of the Capabilities of Cubesats for Earth Observation," Acta Astronautica, Vol. 74, 2012, pp. 50-68, http://systemarchitect.mit.edu/docs/selva12b.pdf. doi: $10.1016 /$ j.actaastro.2011.12.014

-[3] Woellert, K., Ehrenfreund, P., Ricco, A. J., and Hertzfeld, H., "Cubesats: Cost-Effective Science and Technology Platforms for Emerging and Developing Nations," Advances in Space Research, Vol. 47, No. 4, 2011, pp. 663-684. doi:10.1016/j.asr.2010.10.009

[4] Baker, D. N., and Pete Worden, S., "The Large Benefits of SmallSatellite Missions," Transactions American Geophysical Union, Vol. 89, No. 33, Aug. 2008, p. 301. doi:10.1029/2008EO330001 
[5] Moretto, T., and Robinson, R. M., "Small Satellites for Space Weather Research," Space Weather Journal, Vol. 6, No. 5, May 2008, Paper S05007.

[6] Crowley, G., Fish, C. S., Bust, G. S., Swenson, C., Barjatya, A., and Larsen, M. F., "Dynamic Ionosphere Cubesat Experiment (DICE)," Proceedings of the American Geophysical Union (AGU) Fall Meeting, Dec. 2009, p. A6.

[7] Skrobot, G., "ElaNa Educational Launch of Nanosatellite: Enhance Education Through Space Flight," Proceedings of the 25th Annual Small Satellite Conference, Aug. 2011.

[8] Diaz-Aguado, M. F., Ghassemieh, S., Van Outryve, C., Beasley, C., and Schooley, A., "Small Class-D Spacecraft Thermal Design, Test and Analysis-Pharmasat Biological Experiment," Proceedings of the IEEE Aerospace Conference, IEEE Publ., Piscataway, NJ, 2009, pp. 1-9.

[9] Johnson, L., Whorton, M., Heaton, A., Pinson, R., Laue, G., and Adams, C., "Nanosail-D: A Solar Sail Demonstration Mission," Acta Astronautica, Vol. 68, Nos. 5, 6, 2011, pp. 571-575. doi:10.1016/j.actaastro.2010.02.008

[10] Sorgenfrei, M., and Lewis, B., "Biosentinel: Enabling Cubesat-Scale Biological Research Beyond Low Earth Orbit," Proceedings of the Interplanetary Small Satellite Conference, Pasadena, CA, 2014.

[11] Johnson, L., Dervan, J., Frick, A., and Castillo-Rogez, J., "NEA Scout: A CubeSat Architecture for Near Earth Asteroid (NEA) Exploration," Proceedings of the Interplanetary Small Satellite Conference (ISSC), Pasadena, CA, 2014.

[12] Cohe, B., Staehle, R., Banazadeh, P., and Hayne, P., "Lunar FlashLight: A Cubesat Architecture for Deep Space Exploration," Proceedings of the Interplanetary Small Satellite Conference, Pasadena, CA, 2014.

[13] Castillo-Rogez, J., Halatek, L., Murphy, N., Raymond, C., Sherwood, B., Bellardo, J., Cutler, J., Lightsey, G., Klesh, A., and Baker, J., "Inspire: Interplanetary Nanospacecraft Pathfinder in Relevant Environment," Proceedings of the Annual Small Satellite Conference, Logan, UT, Aug. 2013.

[14] Ridley, A., Forbes, J., Cutler, J., Nicholas, A., Thayer, J., Fuller-Rowell, T., Matsuo, T., Bristow, W., Conde, M., Drob, D., Paxton, L., Chappie, S., Osborn, M., Dobbs, M., and Roth, J., "The Armada Mission: Determining the Dynamic and Spatial Response of the Thermosphere/ Ionosphere System to Energy Inputs on Global and Regional Scales," Proceedings of the American Geophysical Union (AGU) Fall Meeting, Dec. 2010, p. A7.

[15] Swenson, C., Larsen, M., Sojka, J., and Fish, C., "CubeSat Constellations for Measurements of High Latitude Energy Input," Proceedings of the American Geophysical Union (AGU) Fall Meeting, Dec. 2009, p. C1584.

[16] Hemmati, H., Jones, D., Klesh, A., Liewer, P., Lazio, J., Wen-Yu Lo, M., Mouroulis, P., Murphy, N., Pingree, P. J., Wilson, T., Anderson, B., Chow, C. C., II, Betts, B., Friedman, L., Puig-Suari, J., Williams, A., Staehle, R. L., Blaney, D., and Svitek, T., "Interplanetary Cubesats: Opening the Solar System to a Broad Community at Lower Cost," Journal of Small Satellites, Vol. 2, No. 1, 2013, pp. 161-186.

[17] Micci, M. M., and Ketsdever, A. D., Micropropulsion for Small Spacecraft, AIAA, Reston, VA, 2000, http://arc.aiaa.org/doi/book/10 $.2514 / 4.866586$.

[18] Rayman, M. D., and Williams, S. N., "Design of the First Interplanetary Solar Electric Propulsion Mission," Journal of Spacecraft and Rockets, Vol. 39, No. 4, 2002, pp. 589-595.

doi: $10.2514 / 2.3848$

[19] Coverstone-Carroll, V., and Williams, S. N., "Mars Missions Using Solar Electric Propulsion," Journal of Spacecraft and Rockets, Vol. 37, No. 1, 2000, pp. 71-77. doi: $10.2514 / 2.3528$

[20] Oh, D. Y., "Evaluation of Solar Electric Propulsion Technologies for Discovery-Class Missions," Journal of Spacecraft and Rockets, Vol. 44, No. 2, 2007, pp. 399-411. doi: $10.2514 / 1.21613$

[21] Molitor, J. H., Schwaiger, L. E., Schollenberger, J. M., Jr., and MacPherson, D., "Solar Electric Propulsion Asteroid Belt Mission," Journal of Spacecraft and Rockets, Vol. 8, No. 6, 1971, pp. 612-617. doi: $10.2514 / 3.59700$

[22] Young, D. T., Nordholt, J. E., Polk, J. E., Henry, M. D., Goldstein, R., Hanley, J. J., Lawrence, D. J., Shappirio, M., Wang, J., and Brinza, D. E., "Deep Space One Investigations of Ion Propulsion Plasma Environment," Journal of Spacecraft and Rockets, Vol. 37, No. 5, 2000 , pp. 545-555. doi: $10.2514 / 2.3608$

[23] Hofer, R. R., Goebel, D. M., Snyder, J. S., and Randolph, T. M., "Simplified Ion Thruster Xenon Feed System for NASA Science Missions," Proceedings of the 31st International Electric Propulsion Conference, Ann Arbor, MI, 2009.
[24] Wilson, R. S., Stuart, J. R., and Howell, K. C., "Automated Design of Propellant-Optimal, Low-Thrust Trajectories for Trojan Asteroid Tours," Journal of Spacecraft and Rockets, Vol. 51, No. 5, 2014, pp. 1631-1647.

[25] Co, T. C., and Black, J. T., "Responsiveness in Low Orbits Using Electric Propulsion," Journal of Spacecraft and Rockets, Vol. 51, No. 3, 2014, pp. 938-945. doi:10.2514/1.A32405

[26] Spangelo, S., and Longmier, B., "Bravosat: Optimizing the Delta-V Capability of a Cubesat Mission with Novel Plasma Propulsion Technology," Proceedings of the Interplanetary Small Satellite Conference, Pasadena, CA, 2013.

[27] Spangelo, S., and Longmier, B., "Optimizing Orbit Transfer Time Using Thrust and Attitude Control for a Cubesat with Interplanetary Applications," Proceedings of the Interplanetary Small Satellite Conference (ISSC), Pasadena, CA, 2014.

[28] Lemmer, K., Kolosa, D., Spangelo, S., and Hudson, J., "Mission Analysis for a Micro RF Ion Thruster for Cubesat Orbital Maneuvers," 50th AIAA/ASME/SAE/ASEE Joint Propulsion Conference, AIAA Paper 2014-3908, July 2014.

[29] Itkoe, Z., "Understanding the Needs of the Cubesat Community," Proceedings of the Summer CubeSat Workshop, Aug. 2011.

[30] Spangelo, S., and Cutler, J., "Analytical Modeling Framework and Applications for Space Communication Networks," Journal of Aerospace Information Systems, Vol. 10, No. 10, 2013, pp. 452-466. doi:10.2514/1.I010086

[31] "Cubesat Design Specification (CDs)," The CubeSat Program, California Polytechnic State Univ., San Luis Obispo, CA, Aug. 2009.

[32] XB1: High Performance 1U Cubesat Bus Specification Sheet, Blue Canyon Technology, Boulder, CO, 2014, http://bluecanyontech.com/ product/xb1/ [retrieved Dec. 2014].

[33] Bering, E. A., Olsen, C. S., Squire, J. P., Ballenger, M. G., Carter, M. D., Cassady, L. D., Chang Daz, F. R., Glover, T. W., Sheehan, J. P., Longmier, B. W., and Ilin, A. V., "Temperature Gradients Due to Adiabatic Plasma Expansion in a Magnetic Nozzle," Plasma Sources Science and Technology, Vol. 23, No. 4, 2014, Article No. 045014.

[34] Longmier, B., and Sheehan, J. P., "Initial Experiments of a New Permanent Magnet Helicon Thruster," Proceedings of the International Electric Propulsion Conference, 2013.

[35] Longmier, B., and Sheehan, J. P., "A New Low Power Plasma Thruster for Nanosatellites," Proceedings of the Joint Propulsion Conference (JPC), 2013.

[36] Longmier, B., Bering, E., Olsen, C., Squire, J., Carter, M., Cassady, L., and Sheehan, J., "Plasma Adiabaticity in a Diverging Magnetic Nozzle," Proceedings of the International Electric Propulsion Conference, 2013.

[37] Longmier, B., and Sheehan, J. P., "A Large Delta-V Plasma Thruster for Nanosatellites, Interplanetary Small-Satellite Conference," Proceedings of the Interplanetary Small Satellite Conference, 2014.

[38] Gergaud, J., Haberkorn, T., and Martinon, P., "Low Thrust MinimumFuel Orbital Transfer: A Homotopic Approach," Journal of Guidance, Control, and Dynamics, Vol. 27, No. 6, 2004, pp. 1046-1060.

[39] "Small Spacecraft Technology State of the Art," NASA TP-2014216648, 2014.

[40] "NNP Series NCP 18650 Specification Sheet," Panasonic Energy Corp., 2014, http://industrial.panasonic.com/lecs/www-data/pdf2/ACI4000/ ACI400 0 CE25.pdf.

[41] "Series: iCAP Specification Sheet," Ioxus, Oneonta, NY, 2014, http://www8.hp.com/h20195/v2/GetDocument.aspx?docname=4AA33586EEE [retrieved Dec. 2014].

[42] Armstrong, T. W., and Colborn, B. L., "Trapped Radiation Model Uncertainties: Model-Data and Model-Model Comparisons," NASA CR-2000-210071, 2000.

[43] Ratliff, M., "Yearly Solar Flare Fluence and Dose," Jet Propulsion Lab. Rept. 5052-96-065, California Inst. of Technology, Pasadena, CA, 1996.

[44] Martin, W., "Satellite Image Collection Optimization," Optical Engineering, Vol. 41, No. 9, 2002, pp. 2083-2087. doi:10.1117/1.1495856

[45] Holbert, K. E., Total Ionizing Dose, Arizona State Univ., Phoenix, AZ, 2007.

[46] Manyak, G., and Bellardo, J., "PolySats Next Generation Avionics Design," Proceedings of the IEEE International Conference on Space Mission Challenges for Information Technology, IEEE Publ., Piscataway, NJ, 2011, pp. 69-76. 
This article has been cited by:

1. Simon Tardivel, Andrew T. Klesh, Stefano Campagnola. Technology Enabling Interplanetary Trajectories for Nanospacecraft. Journal of Spacecraft and Rockets, ahead of print1-11. [Abstract] [Full Text] [PDF] [PDF Plus]

2. Renhe Shi, Li Liu, Teng Long, Jian Liu, Bin Yuan. 2017. Surrogate assisted multidisciplinary design optimization for an all-electric GEO satellite. Acta Astronautica 138, 301-317. [Crossref]

3. Jennifer Hudson, Sara Spangelo, Andrew Hine, Daniel Kolosa, Kristina Lemmer. 2016. Mission Analysis for CubeSats with Micropropulsion. Journal of Spacecraft and Rockets 53:5, 836-846. [Abstract] [Full Text] [PDF] [PDF Plus]

4. Jennifer Hudson, Kristina Lemmer, Andrew Hine. Integration of Micro Electric Propulsion System for CubeSat Orbital Maneuvers . [Citation] [PDF] [PDF Plus] 